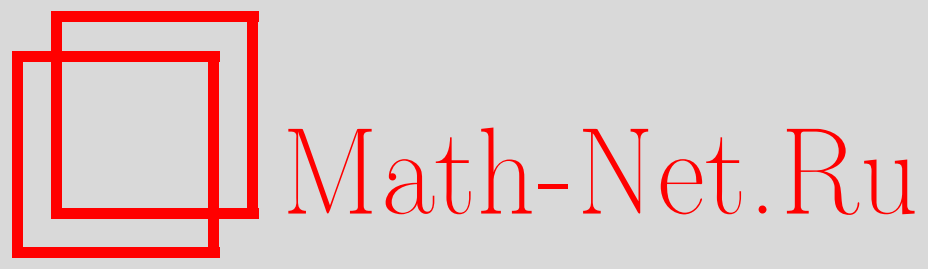

А. А. Зорин, О коммутативности централизатора подалгебры в универсальной обертывающей алгебре, Функи. анализ и его прил., 2009, том 43, выпуск 2, 47-63

DOI: https://doi.org/10.4213/faa2949

Использование Общероссийского математического портала MathNet.Ru подразумевает, что вы прочитали и согласны с пользовательским соглашением

http://www. mathnet.ru/rus/agreement

Параметры загрузки:

IP : 3.89 .185 .249

26 апреля 2023 г., 14:49:11

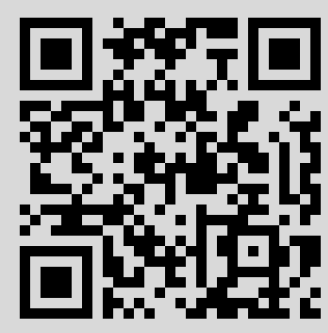


Функциональный анализ и его приложения

2009, т. 43, вып. 2, с. 47-63

УДК 512.816

\title{
О коммутативности централизатора подалгебры в универсальной обертывающей алгебре
}

\author{
(c) 2009. А. А. Зорин
}

\begin{abstract}
Пусть $G$ - редуктивная алгебраическая группа над алгебраически замкнутым полем характеристики 0, a $\mathfrak{h}$ - алгебраическая подалгебра ее касательной алгебры Ли. В работе найдены все подалгебры $\mathfrak{h}$ без нетривиальных характеров, централизаторы $\mathfrak{U}(\mathfrak{g})^{\mathfrak{h}}$ и $P(\mathfrak{g})^{\mathfrak{h}}$ которых в универсальной обертывающей алгебре $\mathfrak{U}(\mathfrak{g})$ и в ассоциированной с ней градуированной алгебре $P(\mathfrak{g})$ коммутативны. Для всех этих подалгебр доказано, что $\mathfrak{U}(\mathfrak{g})^{\mathfrak{h}}=\mathfrak{U}(\mathfrak{h})^{\mathfrak{h}} \otimes \mathfrak{U}(\mathfrak{g})^{\mathfrak{g}}$ и $P(\mathfrak{g})^{\mathfrak{h}}=P(\mathfrak{h})^{\mathfrak{h}} \otimes P(\mathfrak{g})^{\mathfrak{g}}$. Кроме того, получен критерий коммутативности алгебры $\mathfrak{U}(\mathfrak{g})^{\mathfrak{h}}$ в терминах теории представлений.
\end{abstract}

\section{Введение}

Пусть $G$ - редуктивная алгебраическая группа над алгебраически замкнутым полем $k$ характеристики $0, H \subset G$ - ее связная алгебраическая подгруппа, а $\mathfrak{g}$ и $\mathfrak{h}$ - их касательные алгебры Ли. Рассмотрим централизатор $\mathfrak{U}(\mathfrak{g})^{\mathfrak{h}}$ подалгебры $\mathfrak{h}$ в универсальной обертывающей алгебре $\mathfrak{U}(\mathfrak{g})$ алгебры $\mathfrak{g}$. Нас будет интересовать, когда он коммутативен.

Пусть $\rho: \mathfrak{g} \rightarrow \mathfrak{g l}(V)-$ неприводимое представление алгебры $\mathfrak{g}$. Оно продолжается до представления алгебры $\mathfrak{U}(\mathfrak{g})$, которое мы также будем обозначать через $\rho$. Обозначим через $\mathfrak{z} V(\mathfrak{h})$ централизатор подалгебры $\rho(\mathfrak{h})$ в $\mathfrak{g l}(V)$. Следующая теорема, доказательство которой будет приведено в $\S 1$, дает критерий коммутативности централизатора $\mathfrak{U}(\mathfrak{g})^{\mathfrak{h}}$ в терминах теории представлений алгебры $\mathfrak{g}$.

Теорема 1. Алгебра $\mathfrak{U}(\mathfrak{g})^{\mathfrak{h}}$ коммутативна тогда и толъко тогда, когда для любого неприводимого представления $\rho: \mathfrak{g} \rightarrow \mathfrak{g l}(V)$ коммутативна алгебра $\mathfrak{z}_{V}(\mathfrak{h})$.

Для того чтобы алгебра $\mathfrak{U}(\mathfrak{g})^{\mathfrak{h}}$ была коммутативной, необходимо, чтобы централизатор $P(\mathfrak{g})^{\mathfrak{h}}$ алгебры $\mathfrak{h}$ в ассоциированной с $\mathfrak{U}(\mathfrak{g})$ градуированной алгебре $P(\mathfrak{g})$ был коммутативен относительно скобки Пуассона (подробнее см. в $\S 2$ ).

В работе Кнопа [1] было показано, что в случае, когда $\mathfrak{h}$ - редуктивная подалгебра полупростой алгебры Ли $\mathfrak{g}$, алгебра $\mathfrak{U}(\mathfrak{g})^{\mathfrak{h}}$ коммутативна тогда и только тогда, когда коммутативна алгебра $P(\mathfrak{g})^{\mathfrak{h}}$, а также тогда и только тогда, когда она есть тензорное произведение подалгебр $\mathfrak{U}(\mathfrak{g})^{\mathfrak{g}}$ и $\mathfrak{U}(\mathfrak{h})^{\mathfrak{h}}$. В той же работе были найдены все неразложимые пары $(\mathfrak{g}, \mathfrak{h})$, удовлетворяющие этим условиям. Теорема 1 показывает, что в данном случае коммутативность централизатора $\mathfrak{U}(\mathfrak{g})^{\mathfrak{h}}$ эквивалентна тому, что алгебра $\mathfrak{h}$ имеет простой спектр в любом неприводимом представлении алгебры $\mathfrak{g}$.

На пространстве $\mathfrak{g}^{*}$ существует пуассонова структура, алгебра Пуассона которой совпадает с $P(\mathfrak{g})$. Присоединенное представление $H: \mathfrak{g}^{*}$ сохраняет сим- 
плектические слои этой структуры. Если это действие коизотропно на симплектических слоях общего положения (см. определение в §2), то мы будем называть подалгебру $\mathfrak{h} \subset \mathfrak{g}$ и пару $(\mathfrak{g}, \mathfrak{h})$ коизотропными .

Пусть Quot $P(\mathfrak{g})$ - поле частных алгебры $P(\mathfrak{g})$. Алгебра (Quot $P(\mathfrak{g}))^{\mathfrak{h}}$ коммутативна тогда и только тогда, когда алгебра h коизотропна (см. §2). Если группа $H$ не имеет нетривиальных характеров, то оба этих условия равносильны коммутативности алгебры $P(\mathfrak{g})^{\mathfrak{h}}$.

Теорема 2. Пусть $\mathfrak{g}$ - простая алгебра Ли. Собственная подалгебра $\mathfrak{h} \subset \mathfrak{g}$ является коизотропной тогда и только тогда, когда пара $(\mathfrak{g}, \mathfrak{h})$ есть одна из перечисленных ниже:

1) $\left(\mathfrak{s l}_{n+1}, \mathfrak{g l}_{n}\right)(n \geqslant 1),\left(\mathfrak{s o}_{n}, \mathfrak{s o}_{n-1}\right)(n \geqslant 5)$;

2) $\left(\mathfrak{g}, \mathfrak{g}_{\langle v\rangle}\right)$ или $\left(\mathfrak{g}, \mathfrak{g}_{v}\right)$, где $\mathfrak{g}$ - классическая простая алгебра Ли, v - старший вектор ее стандартного представления (изотропный, если $\left.\mathfrak{g}=\mathfrak{s o}_{n}\right),\langle v\rangle-$ прямая, натянутая на $v, \mathfrak{g}_{v} u \mathfrak{g}_{\langle v\rangle}$ - стабилизаторы вектора $v$ и прямой $\langle v\rangle$ соответственно.

3) $\left(\mathfrak{s l}_{n+1}, \mathfrak{p}_{\left(\alpha_{1}, \alpha_{n}\right)}\right)$, где $\mathfrak{p}_{\left(\alpha_{1}, \alpha_{n}\right)}$ - параболическая подалгебра, отвечающая набору простых корней $\left\{\alpha_{1}, \alpha_{n}\right\}$ (определение см. в §5).

4) $\left(\mathfrak{s l}_{3}, \mathfrak{b}\right)$ или $\left(\mathfrak{s l}_{3}, \mathfrak{u}+\left\langle E_{11}-2 E_{22}+E_{33}\right\rangle\right)$, где $\mathfrak{b}-$ борелевская подалгебра в $\mathfrak{s l}_{3}$, a $\mathfrak{u}$ - ее унипотентный радикал.

Пара $(\mathfrak{g}, \mathfrak{h})$ называется разложимой, если существуют такие пары $\left(\mathfrak{g}_{1}, \mathfrak{h}_{1}\right)$ и $\left(\mathfrak{g}_{2}, \mathfrak{h}_{2}\right)$, что $\mathfrak{g}=\mathfrak{g}_{1} \oplus \mathfrak{g}_{2}$ и $\mathfrak{h}=\mathfrak{h}_{1} \oplus \mathfrak{h}_{2}$. В противном случае пара называется неразложимой. Любая коизотропная пара $(\mathfrak{g}, \mathfrak{h})$ представляется в виде суммы коизотропных неразложимых пар.

Теорема 3. Пусть $\mathfrak{g}-$ полупростая алгебра Ли $и \mathfrak{h} \subset \mathfrak{g}-$ ее собственная алгебраическая подалгебра, которая не имеет нетривиальных характеров. Пара $(\mathfrak{g}, \mathfrak{h})$ является коизотропной неразложимой тогда и толъко тогда, когда она есть одна из перечисленных ниже:

1) $\left(\mathfrak{s o}_{n}, \mathfrak{s o}_{n-1}\right), n \geqslant 4$;

2) $\left(\mathfrak{g}, \mathfrak{g}_{v}\right)$, где $\mathfrak{g}$ - классическая простая алгебра Ли, v-стариий вектор ее стандартного представления.

В §3 приведены необходимые факты об индексах алгебр Ли, а в §4 приведена классификация подалгебр Ли коранга не более 4 в алгебре $\mathfrak{s l}_{n+1}$. Эта классификация потребуется нам при доказательстве теоремы 2 в 55 . В 66 доказана теорема 3 .

Обозначим через $Z(\mathfrak{g})$ и $Z(\mathfrak{h})$ центры алгебр Пуассона $P(\mathfrak{g})$ и $P(\mathfrak{h})$ соответственно, а через $\mathfrak{Z}(\mathfrak{g})$ и $\mathfrak{Z}(\mathfrak{h})$ - центры алгебр $\mathfrak{U}(\mathfrak{g})$ и $\mathfrak{U}(\mathfrak{h})$. В $\S 7$ доказана следующая теорема, описывающая строение централизатора коизотропной подалгебры.

Теорема 4. Пусть $(\mathfrak{g}, \mathfrak{h})$ - коизотропная неразложимая пара и $\mathfrak{h}$ не имеет нетривиальных характеров. Тогда

1) $P(\mathfrak{g})^{\mathfrak{h}}=Z(\mathfrak{h}) \otimes Z(\mathfrak{g})$;

2) $\mathfrak{U}(\mathfrak{g})^{\mathfrak{h}}=\mathfrak{Z}(\mathfrak{h}) \otimes \mathfrak{Z}(\mathfrak{g})$, в частности, алгебра $\mathfrak{U}(\mathfrak{g})^{\mathfrak{h}}$ коммутативна.

В работе используются следующие обозначения:

$\mathfrak{a}^{u}$ - унипотентный радикал алгебры Ли $\mathfrak{a}$.

$\mathfrak{a} \bigoplus_{\rho} \mathfrak{n}$ - полупрямая сумма алгебр Ли $\mathfrak{a}$ и $\mathfrak{n}$, где $\rho$ - действие $\mathfrak{a} \rightarrow \mathfrak{g l}(\mathfrak{n})$ (мы будем опускать символ $\rho$, когда из контекста ясно, о каком представлении идет речь). 
$k^{n}$ - линейное пространство над $k$ размерности $n$; так же обозначается $n$-мерная коммутативная алгебра Ли.

$\mathfrak{U}(\mathfrak{a})$ - универсальная обертывающая алгебра алгебры Ли $\mathfrak{a}$.

$P(\mathfrak{a})$ - ассоциированная градуированная алгебра алгебры $\mathfrak{U}(\mathfrak{a})$.

$\mathfrak{U}^{(n)}(\mathfrak{a})-n$-я компонента естественной фильтрации алгебры $\mathfrak{U}(\mathfrak{a})$.

$P^{n}(\mathfrak{a})-n$-я однородная компонента алгебры $P(\mathfrak{a})$.

$\mathfrak{U}^{n}(\mathfrak{a})$ - образ пространства $P^{n}(\mathfrak{a})$ при отображении симметризации.

\section{§1. Доказательство теоремы 1}

Доказательство теоремы 1. Так как представление $\rho$ неприводимо, то $\rho(\mathfrak{U}(\mathfrak{g}))=\mathfrak{g l}(V)$. Пусть $v \in \mathfrak{U}(\mathfrak{g})$ - такой элемент, что $\rho(v) \in \mathfrak{z} V(\mathfrak{h})$. Тогда $[\mathfrak{h}, v] \subset$ Ker $\rho$. Существует такое конечномерное $\mathfrak{g}$-инвариантное подпространство $U \subset$ $\operatorname{Ker} \rho$, что $[\mathfrak{h}, v] \subset U$. Так как $\mathfrak{g}$ редуктивна, то существует $\mathfrak{g}$-инвариантное подпространство $W$, такое, что $\mathfrak{U}(\mathfrak{g})=U \oplus W$. Пусть $v=u+w, u \in U, w \in W$. Тогда $[\mathfrak{h}, w]=[\mathfrak{h}, v]-[\mathfrak{h}, u]$. Так как пространство $U \mathfrak{g}$-инвариантно, то $[\mathfrak{h}, u] \subset U$ и, значит, $[\mathfrak{h}, w] \subset U$. Так как $[\mathfrak{h}, w] \subset W$, то $[\mathfrak{h}, w]=0$, а значит, $w \in \mathfrak{z} \mathfrak{u}(\mathfrak{g})(\mathfrak{h})$. Следовательно, $v \in \mathfrak{z} \mathfrak{U}(\mathfrak{g})(\mathfrak{h})+\operatorname{Ker} \rho$. Таким образом, $\mathfrak{z} V(\mathfrak{h})=\rho(\mathfrak{z} \mathfrak{U}(\mathfrak{g})(\mathfrak{h}))$. Поэтому из коммутативности алгебры $\mathfrak{z} \mathfrak{u}(\mathfrak{g})(\mathfrak{h})$ следует коммутативность алгебры $\mathfrak{z} V(\mathfrak{h})$ для любого неприводимого представления $\rho$.

Если алгебра $\mathfrak{z} \mathfrak{u}(\mathfrak{g})(\mathfrak{h})$ некоммутативна, то существуют $u, v, w \in \mathfrak{z} \mathfrak{u}(\mathfrak{g})(\mathfrak{h})$, такие, что $w=[u, v] \neq 0$. Существует такое представление $\rho: \mathfrak{g} \rightarrow \mathfrak{g l}(V)$, что $\rho(w) \neq 0\left[2\right.$, теорема 2.5.7]. Так как $\rho(u), \rho(v) \in \mathfrak{z}_{V}(\mathfrak{h})$, то алгебра $\mathfrak{z} V(\mathfrak{h})$ некоммутативна.

\section{§2. Геометрический критерий коммутативности алгебры $\boldsymbol{P}(\mathfrak{g})^{\mathfrak{h}}$}

Рассмотрим алгебру Пуассона $P(\mathfrak{g})$. Скобка Пуассона $\{\cdot, \cdot\}$ на $P(\mathfrak{g})$ задается следующим образом. Пусть $\xi \in P^{n}(\mathfrak{g}), \eta \in P^{m}(\mathfrak{g})$. Обозначим через $\hat{\xi} \in$ $\mathfrak{U}^{(n)}(\mathfrak{g}), \hat{\eta} \in \mathfrak{U}^{(m)}(\mathfrak{g})$ некоторые прообразы элементов $\xi$ и $\eta$ в $\mathfrak{U}(\mathfrak{g})$. Тогда $[\hat{\xi}, \hat{\eta}] \in$ $\mathfrak{U}^{(n+m-1)}$. По определению $\{\xi, \eta\}$ есть проекция элемента $[\hat{\xi}, \hat{\eta}]$ на $P^{n+m-1}(\mathfrak{g})$.

Рассмотрим отображение симметризации

$$
\psi: P(\mathfrak{g}) \rightarrow \mathfrak{U}(\mathfrak{g}) .
$$

Пусть $\psi_{n}$ - ограничение этого отображения на однородную компоненту $P^{n}(\mathfrak{g})$. Так как $\psi$ перестановочно с присоединенным представлением алгебры $\mathfrak{h}$, то $\psi_{n}\left(P^{n}(\mathfrak{g})^{\mathfrak{h}}\right)=\mathfrak{U}^{n}(\mathfrak{g})^{\mathfrak{h}}$. Поэтому $P(\mathfrak{g})^{\mathfrak{h}}=\operatorname{gr} \mathfrak{U}(\mathfrak{g})^{\mathfrak{h}}$. Если коммутативна алгебра $\mathfrak{U}(\mathfrak{g})^{\mathfrak{h}}$, то коммутативна и алгебра $P(\mathfrak{g})^{\mathfrak{h}}$.

Рассмотрим коприсоединенное действие $G: \mathfrak{g}^{*}$. На пространстве $\mathfrak{g}^{*}$ существует пуассонова структура, которая на орбитах этого действия задается формой Костанта-Кириллова

$$
\omega\left(\operatorname{ad}^{*}(\xi) \alpha, \operatorname{ad}^{*}(\eta) \alpha\right)=\alpha([\xi, \eta]), \quad \xi, \eta \in \mathfrak{g}, \alpha \in \mathfrak{g}^{*} .
$$

Алгебра Пуассона этой структуры совпадает с $P(\mathfrak{g})$. Симплектическими слоями этой пуассоновой структуры являются орбиты коприсоединенного представления группы Ли $G$. Ограничение алгебры Пуассона на симплектический слой $M$ мы будем обозначать через $P(M)$, а алгебру частных алгебры $P(\mathfrak{g})$ - через Quot $P(\mathfrak{g})$. Ограничение коприсоединенного представления группы $G$ на любой симплектический слой $M$ многообразия $\mathfrak{g}^{*}$ является симплектическим 
действием. Ограничив это действие на подгруппу $H$, мы получим симплектическое действие $H: M$. Коммутативность алгебры (Quot $P(\mathfrak{g}))^{H}$ эквивалентна коммутативности алгебр $(\text { Quot } P(M))^{H}$ для всех симплектических слоев $M$ многообразия $\mathfrak{g}^{*}$.

Корангом действия $H: M$ называется коранг касательного пространства к орбите группы $H$ в точке общего положения слоя $M$. Будем обозначать его чеpeз $\operatorname{cork}_{H} M$. Действие называется коизотропным, если $\operatorname{cork}_{H} M=0$. Известно, что симплектическое действие $H: M$ коизотропно тогда и только тогда, когда алгебра (Quot $P(M))^{H}$ коммутативна. В частности, если действие $H$ : $M$ коизотропно, то алгебра $P(M)^{H}$ коммутативна. Если полиномиальные инварианты группы $H$ разделяют орбиты общего положения на $M$, то верно и обратное: алгебра $P(M)^{H}$ коммутативна, только если действие коизотропно [3, предложение $2.5]$.

Определение 1. Число $\operatorname{cork}_{H} M$, где $M \subset \mathfrak{g}^{*}-$ симплектический слой общего положения, будем называть корангом пары $(\mathfrak{g}, \mathfrak{h})$ или корангом подалгебры $\mathfrak{h}$ в $\mathfrak{g}$. Подалгебру $\mathfrak{h}$ в алгебре $\mathfrak{g}$ будем называть коизотропной, если для симплектического слоя общего положения $M$ действие $H: M$ коизотропно. Coответствующую пару $(\mathfrak{g}, \mathfrak{h})$ также будем называть коизотропной.

Если $\mathfrak{h}_{1}$ и $\mathfrak{h}_{2}$ - подалгебры в $\mathfrak{g}, \mathfrak{h}_{1} \subset \mathfrak{h}_{2}$, и подалгебра $\mathfrak{h}_{1}$ коизотропна, то и $\mathfrak{h}_{2}$ коизотропна.

Отметим, что разложимая пара $(\mathfrak{g}, \mathfrak{h})=\left(\mathfrak{g}_{1} \oplus \mathfrak{g}_{2}, \mathfrak{h}_{1} \oplus \mathfrak{h}_{2}\right)$ является коизотропной тогда и только тогда, когда коизотропны пары $\left(\mathfrak{g}_{1}, \mathfrak{h}_{1}\right)$ и $\left(\mathfrak{g}_{2}, \mathfrak{h}_{2}\right)$. Вся алгебра $\mathfrak{g}$ является коизотропной подалгеброй в себе самой. Таким образом, для классификации всех коизотропных пар $(\mathfrak{g}, \mathfrak{h})$ можно считать, что подалгебра $\mathfrak{h}$ не содержит простых компонент алгебры g. Имеет место следующая

Теорема 5. Пусть алгебра $\mathfrak{g}$ полупроста $и$ подалгебра $\mathfrak{h} \subset \mathfrak{g}$ не содержит простых компонент алгебры $\mathfrak{g}$. Для симплектического слоя $M \subset \mathfrak{g}^{*}$ общего положсения верно равенство

$$
\operatorname{cork}_{H} M=\operatorname{dim} \mathfrak{g}-\operatorname{rk} \mathfrak{g}-\operatorname{dim} \mathfrak{h}-\operatorname{ind} \mathfrak{h} .
$$

Сначала докажем вспомогательную лемму.

Лемма 1. В условиях теоремы 5 стабилизатор точки общего положсения действия $H: M$ конечен.

Доказательство. Можно считать, что $M$ состоит из регулярных полупростых элементов. Стабилизатор $G_{x}$ любой точки $x \in M$ есть некоторый максимальный тор $T_{x}$. Следовательно, симплектический слой общего положения есть $M=G / T$, где $T \subset G$ - максимальный тор.

Действие $G: G / H$ левыми сдвигами имеет конечное ядро неэффективности, так как $\mathfrak{h}$ не содержит простых компонент алгебры $\mathfrak{g}$. Следовательно, и ядро неэффективности действия $T: G / H$ конечно. Поэтому конечен стабилизатор общего положения этого действия [4, п. 7.2], а значит, конечен и стабилизатор общего положения действия $H: G / T$.

Доказательство теоремы 5. Можно считать, что слой $M$ состоит из регулярных полупростых элементов. Тогда $\operatorname{dim} M=\operatorname{dim} \mathfrak{g}-\mathrm{rk} \mathfrak{g}$. Из леммы 1 следует, что коразмерность $\operatorname{codim}_{H}(M)$ орбиты общего положения действия $H: M$ равна $\operatorname{dim} \mathfrak{g}-\mathrm{rk} \mathfrak{g}-\operatorname{dim} \mathfrak{h}$. Обозначим через $\operatorname{def}_{H}(M)$ дефект (размерность ядра) ограничения формы Костанта-Кириллова $\omega$ на касательное пространство 
орбиты общего положения. Имеем

$$
\operatorname{cork}_{H}(M)=\operatorname{codim}_{H}(M)-\operatorname{def}_{H}(M) .
$$

Рассмотрим отображение моментов $\Phi: M \rightarrow \mathfrak{h}^{*}$. Поскольку размерность образа отображения моментов равна размерности орбиты общего положения действия $H: M$ (см. [3]), то образ $\Phi(M)$ плотен в $\mathfrak{h}^{*}$ (лемма 1). Известно, что дефект действия $H: M$ равен коразмерности орбиты общего положения действия $H: \Phi(M)$ [3]. Поскольку $\Phi(M)$ плотен в $\mathfrak{h}^{*}$, то $\operatorname{def}_{H}(M)=\operatorname{ind} \mathfrak{h}$. Из равенства (1) имеем

$$
\operatorname{cork}_{H}(M)=\operatorname{dim} \mathfrak{g}-\operatorname{rk} \mathfrak{g}-\operatorname{dim} \mathfrak{h}-\operatorname{ind} \mathfrak{h} .
$$

Таким образом, подалгебра $\mathfrak{h}$ является коизотропной в $\mathfrak{g}$ тогда и только тогда, когда

$$
\operatorname{dim} \mathfrak{g}-\operatorname{dim} \mathfrak{h}=\operatorname{rk} \mathfrak{g}+\operatorname{ind} \mathfrak{h} .
$$

Следствие. Равенство (*) выполнено тогда и только тогда, когда алгебpa (Quot $P(\mathfrak{g}))^{\mathfrak{h}}$ коммутативна. Если выполнено равенство (*), то алгебра

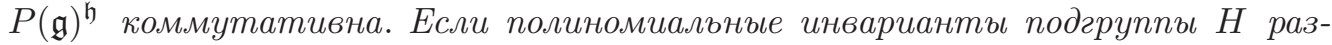
деляют орбиты общего положсения действия $H: \mathfrak{g}^{*}$, то алгебра $P(\mathfrak{g})^{\mathfrak{h}}$ коммутативна тогда и только тогда, когда выполнено равенство (*).

Доказательство. Алгебры инвариантов (Quot $P(\mathfrak{g}))^{\mathfrak{h}}$ и $(\text { Quot } P(\mathfrak{g}))^{H}$ совпадают. Равенство $(*)$ выполнено тогда и только тогда, когда действие на слоях общего положения коизотропно. Последнее равносильно тому, что алгебpa $(\text { Quot } P(M))^{H}$ коммутативна, если $M-$ слой общего положения, т. е. тому, что алгебра $(\text { Quot } P(\mathfrak{g}))^{H}$ коммутативна. Второе утверждение следует из включения Quot $P(\mathfrak{g})^{H} \subset(\text { Quot } P(\mathfrak{g}))^{H}$. Третье - из того, что при указанном дополнительном условии это включение обращается в равенство.

Пример. Рассмотрим алгебру $\mathfrak{g}=\mathfrak{s l}_{n+1}$ и борелевскую подалгебру $\mathfrak{b} \subset \mathfrak{g}$. Алгебра инвариантов $P(\mathfrak{g})^{\mathfrak{b}}$ совпадает с центром алгебры $P(\mathfrak{g})$ и, следовательно, является коммутативной. Известно (см., например, [5]), что ind $\mathfrak{b}=[n / 2]$. Таким образом, при $n \geqslant 3$

$$
\operatorname{dim} \mathfrak{g}-\operatorname{dim} \mathfrak{b}-\operatorname{rk} \mathfrak{g}-\operatorname{ind} \mathfrak{b}=\frac{n(n-1)}{2}-\left[\frac{n}{2}\right] \neq 0
$$

и равенство $(*)$ не выполнено. Следовательно, в общем случае из коммутативности алгебры $P(\mathfrak{g})^{\mathfrak{h}}$ не следует коизотропность.

Пусть $H$ - связная редуктивная подгруппа полупростой алгебраической группы Ли G. В работе Кнопа [1] для этого случая найдены все собственные неразложимые пары $(\mathfrak{g}, \mathfrak{h})$, такие, что алгебра $\mathfrak{U}(\mathfrak{g})^{\mathfrak{h}}$ коммутативна. Это пары $\left(\mathfrak{s l}_{n}, \mathfrak{g l}_{n-1}\right), n \geqslant 2$, и $\left(\mathfrak{s o}_{n}, \mathfrak{s o}_{n-1}\right), n \geqslant 4$. Действие подгруппы $H \subset G$ на $M=G / T$ всегда стабильно [6]; поэтому $H$-орбиты общего положения действия $H: \mathfrak{g}^{*}$ разделяются полиномиальными инвариантами [4]. Следовательно, указанные пары являются коизотропными.

Интересно найти все коизотропные нередуктивные подалгебры $\mathfrak{h}$ полупростых алгебр Ли. В §5 описаны все такие подалгебры простых алгебр Ли, а в $\S 6$ - все коизотропные подалгебры, не имеющие нетривиальных характеров, в полупростых алгебрах Ли. Отсутствие нетривиальных характеров равносильно 
тому, что подалгебра Леви алгебры $\mathfrak{h}$ полупроста. В этом случае полиномиальные инварианты представления $H: \mathfrak{g}^{*}$ разделяют орбиты группы $H$ на симплектическом слое общего положения. Следовательно, при данном ограничении найдены и все такие пары $(\mathfrak{g}, \mathfrak{h})$, что алгебра $P(\mathfrak{g})^{\mathfrak{h}}$ коммутативна.

\section{§3. Некоторые сведения об индексах алгебр Ли}

Определение 3. Пусть $\mathfrak{g}$ - алгебра Ли. Рассмотрим линейное представление $\rho: \mathfrak{g} \rightarrow \mathfrak{g l}(V)$. Число $\operatorname{dim} V-\max _{\xi \in V^{*}} \operatorname{dim} \mathfrak{g} \xi$ называется его индексом. Будем обозначать его через $\operatorname{ind}(\mathfrak{g}, V)$ или $\operatorname{ind}(\rho, V)$. Элемент $\xi \in V^{*}$ называется регулярным (или $\rho$-регулярным), если пространство $\mathfrak{g} \xi$ имеет максимальную размерность. Индекс присоединенного представления называется индексом алгебры Ли $\mathfrak{g}$ и обозначается через ind $\mathfrak{g}$.

Пусть $\mathfrak{g}-$ редуктивная алгебра Ли и $\mathfrak{p} \subset \mathfrak{g}$ - ее параболическая подалгебра. Известно ([5], [7]), что ind $\mathfrak{g}=\mathrm{rk} \mathfrak{g}$ и ind $\mathfrak{p} \leqslant \mathrm{rk} \mathfrak{g}$. Если $\mathfrak{p}$ - максимальная параболическая подалгебра в $\mathfrak{g l}_{n}$ и ее подалгебра Леви есть $\mathfrak{g l}_{n_{1}} \oplus \mathfrak{g l}_{n_{2}}, n_{1}+n_{2}=n$, то ind $\mathfrak{p}=\operatorname{gcd}\left(n_{1}, n_{2}\right)$ [8].

Лемма 2. Пусть $\mathfrak{h}-$ подалгебра алгебры $\mathfrak{g}$. Тогда ind $\mathfrak{g} \leqslant \operatorname{ind} \mathfrak{h}+(\operatorname{dim} \mathfrak{g}-$ $\operatorname{dim} \mathfrak{h})$.

Доказательство. Пусть $\xi_{0} \in \mathfrak{h}^{*}$ - регулярный элемент коприсоединенного представления алгебры $\mathfrak{h}$. Тогда $\operatorname{dim} \mathfrak{h} \xi_{0}=\operatorname{dim} \mathfrak{h}-\operatorname{ind} \mathfrak{h}$. Продолжим этот элемент до линейной формы $\xi \in \mathfrak{g}^{*}$. Тогда $\operatorname{dim} \mathfrak{g} \xi \geqslant \operatorname{dim} \mathfrak{h} \xi \geqslant \operatorname{dim} \mathfrak{h} \xi_{0}$. Следовательно,

$$
\text { ind } \mathfrak{g} \leqslant \operatorname{dim} \mathfrak{g}-\operatorname{dim} \mathfrak{g} \xi \leqslant \operatorname{dim} \mathfrak{g}-\operatorname{dim} \mathfrak{h} \xi_{0} \leqslant \operatorname{dim} \mathfrak{g}-\operatorname{dim} \mathfrak{h}+\text { ind } \mathfrak{h} .
$$

Пусть задано некоторое линейное представление $\rho: \mathfrak{s} \rightarrow \mathfrak{g l}(V)$ алгебры Ли $\mathfrak{s}$. Тогда на пространстве $\mathfrak{s} \oplus V$ существует каноническая структура алгебры Ли, а именно, структура полупрямого произведения алгебры $\mathfrak{s}$ и коммутативного идеала $V$. На алгебре $\mathfrak{g}=\mathfrak{s} \oplus_{\rho} V$ имеется $\mathbb{Z}$-градуировка $\mathfrak{g}=\mathfrak{g}(0) \oplus \mathfrak{g}(1)$, где $\mathfrak{g}(0)=\mathfrak{s}, \mathfrak{g}(1)=V$. Двойственное пространство $\mathfrak{g}^{*}$ можно отождествить с пространством $\mathfrak{s}^{*} \oplus V^{*}=\mathfrak{g}^{*}(0) \oplus \mathfrak{g}^{*}(-1)$. Имеет место следующая

Теорема 6 (Раис [9]). Пусть $\xi \in \mathfrak{g}^{*}(-1)$ есть $\rho$-регулярный элемент, такой, что для некоторого $\eta \in \mathfrak{g}^{*}(0)$ элемент $\eta+\xi \in \mathfrak{g}^{*}(0) \oplus \mathfrak{g}^{*}(-1)$ такэе регулярен. Тогда

$$
\text { ind } \mathfrak{g}=\operatorname{ind}(\mathfrak{g}(0), \mathfrak{g}(1))+\operatorname{ind} \mathfrak{g}(0)_{\xi},
$$

где $\mathfrak{g}(0)_{\xi}$ - стабилизатор элемента $\xi$ в алгебре $\mathfrak{g}(0)$.

Отметим, что множество векторов $\xi$, которые удовлетворяют условию теоремы, плотно в $\mathfrak{g}^{*}(-1)$.

Д. И. Панюшевым было получено обобщение теоремы Раиса для градуированных алгебр Ли вида $\mathfrak{g}=\mathfrak{g}(0) \oplus \mathfrak{g}(1) \oplus \mathfrak{g}(2)$. Положим $\mathfrak{g}^{*}(-j)=\operatorname{Ann}\left(\bigoplus_{i \neq j} \mathfrak{g}(i)\right)$ $\simeq \mathfrak{g}(j)^{*}$. Тогда $\mathfrak{g}^{*}=\mathfrak{g}^{*}(0) \oplus \mathfrak{g}^{*}(-1) \oplus \mathfrak{g}^{*}(-2)$.

Теорема 7 [5]. Предположим, что существует $\mathfrak{g}(0)$-регулярный элемент $\xi \in \mathfrak{g}^{*}(-2)$, такой, что $\mathfrak{g}_{\xi} \cap \mathfrak{g}(1)=\{0\}$. Тогда

$$
\text { ind } \mathfrak{g}=\operatorname{ind}(\mathfrak{g}(0) \oplus \mathfrak{g}(2))=\operatorname{ind}(\mathfrak{g}(0), \mathfrak{g}(2))+\operatorname{ind} \mathfrak{g}(0)_{\xi_{0}},
$$

где $\xi_{0}-$ подходящий регулярный элемент из $\mathfrak{g}^{*}(-2)$. 
Мы будем использовать теоремы 6 и 7 для нахождения индексов некоторых алгебр Ли.

Лемма 3. Пусть $\mathfrak{g}$ - классическая простая алгебра Ли, а v-старший вектор в ее стандартном представлении. Имеют место следующие равенства:

(a) ind $\mathfrak{g}_{v}=1$, еслu $\mathfrak{g}=\mathfrak{s l}_{n} ;$ ind $\mathfrak{g}_{v}=0$, еслu $\mathfrak{g}=\mathfrak{g l}_{n}$;

(b) ind $\mathfrak{g}_{v}=\left[\frac{n-1}{2}\right]$, если $\mathfrak{g}=\mathfrak{s o}_{n}$;

(c) ind $\mathfrak{g}_{v}=n$, ecлu $\mathfrak{g}=\mathfrak{s p}_{2 n}$;

(d) ind $\left[\mathfrak{p}_{\left(\alpha_{1}, \alpha_{n-1}\right)}, \mathfrak{p}_{\left(\alpha_{1}, \alpha_{n-1}\right)}\right]=n-2$, если $\mathfrak{p}_{\left(\alpha_{1}, \alpha_{n-1}\right)} \subset \mathfrak{s l}_{n}-$ параболическая подалгебра, отвечающая набору корней $\left\{\alpha_{1}, \alpha_{n-1}\right\}$ (определение см. в 5 );

(e) ind $\mathfrak{h}=3$, если $\mathfrak{h}=\mathfrak{s l}_{2} \oplus_{\rho}\left(V_{1} \otimes V_{2}\right), V_{1}=V_{2}=k^{2}$, где $\rho$ есть произведение стандартного представления на первом множителе и тривиального представления на втором.

Доказательство. (а) Алгебра $\mathfrak{h}=\mathfrak{s} \oplus_{\rho} k^{n-1}-$ полупрямая сумма алгебры $\mathfrak{s}=\mathfrak{s l}_{n-1}$ и идеала $k^{n-1}$. Действие $\rho$ алгебры $\mathfrak{s}$ на идеале $k^{n-1}$ есть стандартное представление алгебры $\mathfrak{s l}_{n-1}$. Оно транзитивно на множестве ненулевых векторов. Можно применить теорему Раиса для ненулевого вектора $\xi \in k^{n-1}$ общего положения. Получаем

$$
\text { ind } \mathfrak{h}=\operatorname{ind}\left(\mathfrak{s l}_{n-1}\right)_{v}+\operatorname{ind}\left(\mathfrak{s l}_{n-1}, k^{n-1}\right) .
$$

Так как $\operatorname{ind}\left(\mathfrak{s l}_{n-1}, k^{n-1}\right)=0$, то по индукции ind $\mathfrak{h}=\left(\mathfrak{s l}_{2}\right)_{v}=1$. Второе равенство получается аналогичным образом.

(b) Как и в п. (а), имеем разложение $\mathfrak{h}=\mathfrak{s o}_{n-2} \oplus_{\rho} k^{n-2}$ и действие $\rho$ алгебры $\mathfrak{s o}_{n-2}$ транзитивно на множестве неизотропных одномерных подпространств в $k^{n-2}$. Поэтому ind $\left(\mathfrak{s o}_{n-2}, k^{n-2}\right)=1$. Применяя теорему Раиса для невырожденного вектора $\xi \in k^{n-2}$ общего положения, получаем

$$
\text { ind } \mathfrak{h}=\text { ind } \mathfrak{s o}_{n-3}+\operatorname{ind}\left(\mathfrak{s o}_{n-2}, k^{n-2}\right) \text {. }
$$

Если $n=2 m$, то ind $\mathfrak{h}=m-1$. Если $n=2 m+1$, то ind $\mathfrak{h}=m$.

(c) На алгебре $\mathfrak{h}$ существует следующая $\mathbb{Z}$-градуировка: $\mathfrak{h}=\mathfrak{h}(0) \oplus \mathfrak{h}(1) \oplus \mathfrak{h}(2)$, где $\mathfrak{h}(0)=\mathfrak{s p}_{2 n-2}, \mathfrak{h}(1) \oplus \mathfrak{h}(2)-$ нильпотентный идеал, изоморфный алгебре Гейзенберга, и $\mathfrak{h}(2)$ - его центр. Алгебра $\mathfrak{h}(0)$ действует тривиально на пространстве $\mathfrak{h}^{*}(-2)$. Для любого вектора $\xi \in \mathfrak{h}^{*}(-2)$ выполняется равенство $\mathfrak{h}_{\xi} \cap \mathfrak{h}(1)=\{0\}$. Поэтому можно применить теорему 7 для любого вектора $\xi \in \mathfrak{h}^{*}(-2)$. Тогда

$$
\text { ind } \mathfrak{h}=\operatorname{ind} \mathfrak{h}(0)_{\xi}+\operatorname{ind}(\mathfrak{h}(0), \mathfrak{h}(2))=\text { ind } \mathfrak{s p}_{2 n-2}+1=n \text {. }
$$

(d) Как и в п. (с), на алгебре $\mathfrak{h}=\mathfrak{p}_{\left(\alpha_{1}, \alpha_{n-1}\right)}$ существует $\mathbb{Z}$-градуировка, $\mathfrak{h}=$ $\mathfrak{h}(0) \oplus \mathfrak{h}(1) \oplus \mathfrak{h}(2)$, где $\mathfrak{h}(0)=\mathfrak{s l}_{2 n-2}, \mathfrak{h}(1) \oplus \mathfrak{h}(2)$ есть нильпотентный идеал, изоморфный алгебре Гейзенберга, и $\mathfrak{h}(2)$ - его центр. Применяя теорему 7 для вектора $\xi \in \mathfrak{h}^{*}(-2)$, получаем

$$
\text { ind } \mathfrak{h}=\operatorname{ind} \mathfrak{h}(0)_{\xi}+\operatorname{ind}(\mathfrak{h}(0), \mathfrak{h}(2))=\text { ind } \mathfrak{s l}_{n-2}+1=n-2 \text {. }
$$

(е) Алгебра $\mathfrak{s l}_{2}$ действует локально транзитивно на первом множителе и тривиально на втором. Применяя теорему Раиса для точки $(\xi, \eta) \in V_{1} \otimes V_{2}$ общего положения, получаем при $\xi \neq 0$, что

$$
\text { ind } \mathfrak{h}=\operatorname{ind}\left(\mathfrak{s l}_{2}\right)_{\xi}+\operatorname{ind}\left(\mathfrak{s l}_{2}, V\right)=1+2=3 .
$$




\section{§4. Редуктивные подалгебры малых корангов в алгебре $\mathfrak{s l}_{n+1}$}

В этом параграфе мы найдем все редуктивные подалгебры корангов 2 и 4 в $\mathfrak{s l}_{n+1}$.

Пусть $\mathfrak{g}$ - классическая алгебра Ли и $\mathfrak{h}$ - ее собственная редуктивная подалгебра. Тогда формула $(*)$ принимает вид

$$
\operatorname{cork}_{\mathfrak{g}} \mathfrak{h}=\operatorname{dim} \mathfrak{g}-\operatorname{dim} \mathfrak{h}-\operatorname{rk} \mathfrak{g}-\operatorname{rk} \mathfrak{h} .
$$

При этом rk $\mathfrak{h} \leqslant \mathrm{rk} \mathfrak{g}$, т. е.

$$
\operatorname{cork}_{\mathfrak{g}} \mathfrak{h} \geqslant \operatorname{dim} \mathfrak{g}-\operatorname{dim} \mathfrak{h}-2 \operatorname{rk} \mathfrak{g} .
$$

Отметим, что если $\mathfrak{h}_{1} \subsetneq \mathfrak{h}_{2}$, то

$$
\operatorname{cork}_{\mathfrak{g}} \mathfrak{h}_{1}-\operatorname{cork}_{\mathfrak{g}} \mathfrak{h}_{2}=\operatorname{dim} \mathfrak{h}_{2}-\operatorname{dim} \mathfrak{h}_{1}+\operatorname{rk} \mathfrak{h}_{2}-\operatorname{rk} \mathfrak{h}_{1} .
$$

В частности, $\operatorname{cork}_{\mathfrak{g}} \mathfrak{h}_{1}>\operatorname{cork}_{\mathfrak{g}} \mathfrak{h}_{2}$.

Пусть $\mathfrak{g}=\mathfrak{s l}_{n+1}$ и коранг подалгебры $\mathfrak{h}$ не превосходит 4. Тогда из равенства (2) следует, что $\operatorname{dim} \mathfrak{h} \geqslant n^{2}-4$. Можно рассматривать вложение алгебры $\mathfrak{h}$ в $\mathfrak{g}=\mathfrak{s l}_{n+1}$ как ее представление $\rho$ в пространстве $V$ размерности $n+1$. Будем называть вложение (подалгебру $\mathfrak{h}$ ) максимальным (максимальной), если не существует редуктивной алгебры $\mathfrak{h}_{0}$, такой, что $\mathfrak{h} \subsetneq \mathfrak{h}_{0} \subsetneq \mathfrak{g}$. Пусть представление $\rho$ приводимо: $V=V_{1} \oplus V_{2}, \operatorname{dim} V_{1}=n_{1}, \operatorname{dim} V_{2}=n_{2}, n_{1}+n_{2}=n+1$. Соответствующее вложение будем также называть приводимым. Тогда $\mathfrak{h} \subset\left(\mathfrak{g l}_{n_{1}} \oplus \mathfrak{g l}_{n_{2}}\right) \cap \mathfrak{s l}_{n+1}$. Следовательно, $\operatorname{dim} \mathfrak{h} \leqslant n_{1}^{2}+n_{2}^{2}-1$, откуда вытекает, что $\left(n-n_{1}\right)\left(1-n_{1}\right)+2 \geqslant 0$. Получаем, что существуют следующие максимальные приводимые вложения редуктивных алгебр коранга не больше 4 в $\mathfrak{s l}_{n+1}$ :

(i) $\mathfrak{g l}_{n} \subset \mathfrak{s l}_{n+1}, \operatorname{cork}_{\mathfrak{g}} \mathfrak{h}=0$;

(ii) $\left(\mathfrak{g l}_{2} \oplus \mathfrak{g l}_{2}\right) \cap \mathfrak{s l}_{4} \subset \mathfrak{s l}_{4}, \operatorname{cork}_{\mathfrak{g}} \mathfrak{h}=2$;

(iii) $\left(\mathfrak{g l}_{2} \oplus \mathfrak{g l}_{3}\right) \cap \mathfrak{s l}_{5} \subset \mathfrak{s l}_{5}, \operatorname{cork}_{\mathfrak{g}} \mathfrak{h}=4$.

Пары с немаксимальными подалгебрами $\mathfrak{h}$ коранга не более 4 должны быть вложены в одну из максимальных пар (i)-(iii). В случае (iii) таких пар не существует. В случае (i) таких пар две:

(iv) $\mathfrak{s l}_{n} \subset \mathfrak{s l}_{n+1}, \operatorname{cork}_{\mathfrak{g}} \mathfrak{h}=2$;

(v) $\mathfrak{t}_{2} \subset \mathfrak{s l}_{3}$, где $\mathfrak{t}_{2}-$ максимальный двумерный тор, $\operatorname{cork}_{\mathfrak{g}} \mathfrak{h}=2$.

В случае (ii) также имеются две пары:

(vi) $\mathfrak{s l}_{2} \oplus \mathfrak{s l}_{2} \subset \mathfrak{s l}_{4}, \operatorname{cork}_{\mathfrak{g}} \mathfrak{h}=4$;

(vii) $\mathfrak{g l}_{2} \oplus \mathfrak{t}_{1} \subset \mathfrak{s l}_{4}$, где $\mathfrak{t}_{1}$ - одномерный тор, $\operatorname{cork}_{\mathfrak{g}} \mathfrak{h}=4$.

Если представление алгебры $\mathfrak{h}$ в пространстве $V$ неприводимо, то $\operatorname{dim} \mathfrak{h}>$ $\operatorname{dim} V$ при $n \geqslant 3$. Известен список всех представлений простых алгебр со свойством $\operatorname{dim} \mathfrak{h}>\operatorname{dim} V$ [10], и прямая проверка показывает, что ни для одного из них не выполнено условие $(* *)$. При $n=1$ в $\mathfrak{g}=\mathfrak{s l}_{2}$ есть единственная редуктивная подалгебра $\mathfrak{t}_{1}$, и этот случай входит в рассмотренную выше серию $\mathfrak{g l}_{n} \subset \mathfrak{s l}_{n+1}$. При $n=2$ алгебра $\mathfrak{h}$ обладает неприводимым представлением в пространстве размерности 3 . Алгебры, удовлетворяющие этому условию, - это $\mathfrak{t}_{2}, \mathfrak{s l}_{2}, \mathfrak{g l}_{2}$. Все получающиеся пары были уже найдены выше.

Пусть подалгебра $\mathfrak{h}$ не является простой: $\mathfrak{h}=\mathfrak{h}_{1} \oplus \mathfrak{h}_{2}$. Тогда $V$ можно представить в следующем виде: $V=V_{1} \otimes V_{2}$, где $V_{1}, V_{2}$ - такие пространства, что представления $\rho_{i}: \mathfrak{h}_{i} \rightarrow \mathfrak{g l}\left(V_{i}\right), i=1,2$, точны. Положим $n_{1}=\operatorname{dim} V_{1}, n_{2}=\operatorname{dim} V_{2}$, 
$n_{1} \leqslant n_{2}$. Тогда $\operatorname{dim} V=n_{1} n_{2}, \operatorname{dim} \mathfrak{h}_{1} \leqslant n_{1}^{2}, \operatorname{dim} \mathfrak{h}_{2} \leqslant n_{2}^{2}$. Из условия (3) следует, чTо

$$
n_{1}^{2}+n_{2}^{2} \geqslant\left(n_{1} n_{2}-1\right)^{2}-4 .
$$

При $n_{1}, n_{2} \geqslant 2$ имеем $\left(n_{1} n_{2}\right)^{2} \geqslant 2 n_{1}^{2}+2 n_{2}^{2}$. Следовательно, $\left(n_{1}-n_{2}\right)^{2} \leqslant 3$ и $n_{1}=n_{2}$ или $n_{1}=n_{2}-1$. Легко видеть, что в обоих случаях неравенство (4) не выполнено. Если же $n_{1}=1$, то подалгебра $\mathfrak{h}_{1}$ является одномерным тором и действует на пространстве $V$ скалярно, а значит, $\mathfrak{h}_{1}=0$. Таким образом, список пар коранга не более 4 исчерпывается парами (i)-(vii).

\section{§5. Нередуктивные коизотропные подалгебры в простых алгебрах Ли}

Найдем сначала все коизотропные параболические подалгебры $\mathfrak{p}$ простых алгебр Ли. Пусть $\left\{\alpha_{i_{1}}, \ldots, \alpha_{i_{k}}\right\} \in \Pi$ - набор простых корней простой алгебры Ли $\mathfrak{g}$ и $\mathfrak{p}=\mathfrak{p}_{\left(\alpha_{i_{1}}, \ldots, \alpha_{i_{k}}\right)}$ - ее параболическая подалгебра, отвечающая набору $\left\{\alpha_{i_{1}}, \ldots, \alpha_{i_{k}}\right\}$, а именно, подалгебра, содержащая корневые подпространства, отвечающие отрицательным корням, в разложении которых на простые корни не содержится ни один из корней $\alpha_{i_{1}}, \ldots, \alpha_{i_{k}}$ Известно [7], что ind $\mathfrak{p} \leqslant \mathrm{rkg}$. Поэтому для коизотропности подалгебры $\mathfrak{p}$ необходимо, чтобы

$$
\operatorname{dim} \mathfrak{g}-\operatorname{dim} \mathfrak{p} \leqslant 2 \operatorname{rk} \mathfrak{g} .
$$

Левая часть этого неравенства равна количеству положительных корней алгебры $\mathfrak{g}$, в разложении которых на простые корни встречается хотя бы один из корней $\alpha_{i_{1}}, \ldots, \alpha_{i_{k}}$. Легко видеть, что необходимое равенство $(*)$ выполняется (с точностью до симметрии схемы Дынкина) в серии $A_{n}$ только для наборов корней $\left\{\alpha_{1}\right\},\left\{\alpha_{2}\right\}$ и $\left\{\alpha_{1}, \alpha_{n}\right\}$, в сериях $B_{n}, C_{n}, D_{n}$ - только для набора $\left\{\alpha_{1}\right\}$ и никогда не выполняется для особых алгебр Ли. Серии $B, C$, $D$ дают пары $\left(\mathfrak{s o}_{n},\left(\mathfrak{s o}_{n}\right)_{\langle v\rangle}\right),\left(\mathfrak{s p}_{n},\left(\mathfrak{s p}_{n}\right)_{\langle v\rangle}\right)$, где $v$ - старший вектор стандартного представления. Для этих пар индексы подалгебр известны [5]. Прямая проверка равенства $(*)$ показывает, что все эти пары являются коизотропными. Аналогично, серии $A_{n}$ и корню $\alpha_{1}$ соответствует пара $\left(\mathfrak{s l}_{n+1},\left(\mathfrak{s l}_{n+1}\right)_{\langle v\rangle}\right)$, где $v-$ старший вектор стандартного представления, и она также является коизотропной. Для $\left\{\alpha_{2}\right\}$ имеем $\operatorname{dim} \mathfrak{g}-\operatorname{dim} \mathfrak{p}_{\alpha_{2}}-\operatorname{rk} \mathfrak{g}=n-2$. Из результата Элашвили [8] следует, что ind $\mathfrak{p}_{\alpha_{2}}=1$, когда $n$ нечетно, и ind $\mathfrak{p}_{\alpha_{2}}=0$, когда $n$ четно. Поэтому равенство выполнено только при $n=2,3$. При $n=2$ этот случай эквивалентен случаю первого простого корня, а при $n=3-$ случаю $\left(\mathfrak{s o}_{6},\left(\mathfrak{s o}_{6}\right)_{\langle v\rangle}\right)$. Индекс алгебры $\mathfrak{p}_{\left(\alpha_{1}, \alpha_{n}\right)}$ равен $n-1$ [5], и она также удовлетворяет равенству (*) и, следовательно, является коизотропной. Таким образом, существуют только следующие коизотропные пары $(\mathfrak{g}, \mathfrak{h})$ с параболической подалгеброй $\mathfrak{h}:\left(\mathfrak{s l}_{n},\left(\mathfrak{s l}_{n}\right)_{\langle v\rangle}\right)$, $\left(\mathfrak{s o}_{n},\left(\mathfrak{s o}_{n}\right)_{\langle v\rangle}\right),\left(\mathfrak{s p}_{n},\left(\mathfrak{s p}_{n}\right)_{\langle v\rangle}\right),\left(\mathfrak{s l}_{n+1}, \mathfrak{p}_{\left(\alpha_{1}, \alpha_{n}\right)}\right)$.

Пусть $\mathfrak{h}$ - произвольная коизотропная нередуктивная подалгебра алгебры $\mathfrak{g}$. Как известно [11], она вкладывается правильным образом в некоторую параболическую подалгебру $\mathfrak{p}$, которая также должна быть коизотропной в $\mathfrak{g}$. Зафиксируем разложения Леви $\mathfrak{h}=\mathfrak{k} \oplus \mathfrak{h}^{u}$ и $\mathfrak{p}=\mathfrak{l} \oplus \mathfrak{p}^{u}$. Тогда $\mathfrak{h}^{u} \subset \mathfrak{p}^{u}$, и можно считать, что $\mathfrak{k} \subset \mathfrak{l}$. Имеем $\mathfrak{l}=\mathfrak{s} \oplus \mathfrak{z}$, где $\mathfrak{z}$ - центр и $\mathfrak{s}=[\mathfrak{l}, \mathfrak{l}]-$ полупростая часть алгебры ‥ Из леммы 2 следует, что

$$
\text { ind } \mathfrak{h} \leqslant \operatorname{ind} \mathfrak{k}+\operatorname{dim} \mathfrak{h}^{u} \text {, }
$$


а также $\mathrm{rk} \mathfrak{g}=\operatorname{rk} \mathfrak{l}$ и $\operatorname{dim} \mathfrak{g}-\operatorname{dim} \mathfrak{h}=\operatorname{dim} \mathfrak{l}-\operatorname{dim} \mathfrak{k}+2 \operatorname{dim} \mathfrak{p}^{u}-\operatorname{dim} \mathfrak{h}^{u}$. Поэтому из равенства $(*)$ получаем, что

$$
\operatorname{dim} \mathfrak{l}-\operatorname{dim} \mathfrak{k}+2\left(\operatorname{dim} \mathfrak{p}^{u}-\operatorname{dim} \mathfrak{h}^{u}\right)-\operatorname{rk} \mathfrak{l}-\operatorname{rk} \mathfrak{k} \leqslant 0
$$

и, значит,

$$
\operatorname{dim} \mathfrak{l}-\operatorname{dim} \mathfrak{k}-\operatorname{rk} \mathfrak{l}-\operatorname{rk} \mathfrak{k} \leqslant 0 .
$$

Если $\mathfrak{k} \subset \mathfrak{s}$ (это верно, в частности, когда алгебра $\mathfrak{k}$ не имеет характеров, т. е. полупроста), то

$$
\operatorname{dim} \mathfrak{s}-\operatorname{dim} \mathfrak{k}-\operatorname{rk} \mathfrak{s}-\operatorname{rk} \mathfrak{k} \leqslant 0 .
$$

Отметим, что в этом случае $\mathfrak{h} \subset \mathfrak{q}=[\mathfrak{p}, \mathfrak{p}]$. Следовательно, подалгебра $\mathfrak{q}$ также является коизотропной в $\mathfrak{g}$. Индексы коммутантов коизотропных параболических подалгебр вычислены в лемме 3, пп. (a)-(d). Легко видеть, что коммутанты являются коизотропными подалгебрами во всех случаях, кроме случая $\left(\mathfrak{s l}_{n},\left(\mathfrak{q}_{\left(\alpha_{1}, \alpha_{n}\right)}\right)\right)$. Коммутант алгебры $\mathfrak{g}_{\langle v\rangle}$, где $\mathfrak{g}-$ классическая простая алгебра Ли, есть $\mathfrak{g}_{v}$. Таким образом, пары $\left(\mathfrak{s l}_{n},\left(\mathfrak{s l}_{n}\right)_{v}\right),\left(\mathfrak{s o}_{n},\left(\mathfrak{s o}_{n}\right)_{v}\right),\left(\mathfrak{s p}_{n},\left(\mathfrak{s p}_{n}\right)_{v}\right)$ являются коизотропными; при этом подалгебры $\mathfrak{h}$ в этих парах не имеют нетривиальных характеров. Пара $(\mathfrak{g}, \mathfrak{h})$ должна содержаться в одной из таких пар.

Если $\mathfrak{k}$ не содержит простых компонент подалгебры $\mathfrak{s}$, то по теореме 5 из (9) следует, что $\mathfrak{k}$ - коизотропная подалгебра в $\mathfrak{s}$ и в неравенстве (9) достигается равенство. Следовательно, равенства достигаются и в (6) и (7). Так как $\mathfrak{s}-$ классическая простая алгебра Ли или $\mathfrak{s o}_{4}$, то из классификации Кнопа, приведенной в конце $\S 2$, получаем, что возможны следующие случаи: $(\mathfrak{s}, \mathfrak{k})=\left(\mathfrak{s l}_{n}, \mathfrak{g l}_{n-1}\right)$ или $(\mathfrak{s}, \mathfrak{k})=\left(\mathfrak{s o}_{n}, \mathfrak{s o}_{n-1}\right)$.

Пусть $(\mathfrak{s}, \mathfrak{k})=\left(\mathfrak{s o}_{n}, \mathfrak{s o}_{n-1}\right)$. Из неравенства (7) следует, что $\mathfrak{h}^{u}=\mathfrak{p}^{u}$, причем $\mathfrak{p}^{u}=k^{n}$ - коммутативная алгебра Ли. Пространство $k^{n}$ разлагается на два неприводимых подпространства относительно действия $\mathfrak{s o}_{n-1}: k^{n}=k^{n-1} \oplus k$. Таким образом, $\mathfrak{h}=\mathfrak{s o}_{n-1} \oplus\left(k^{n-1} \oplus k\right) \subset \mathfrak{s o}_{n+1}$. Из леммы 3 , п. (b), вытекает, что

$$
\text { ind } \mathfrak{h}=\operatorname{ind}\left(\mathfrak{s o}_{n-1} \oplus k^{n-1}\right)+1=\left[\frac{n-1}{2}\right]+1 .
$$

Получаем, что ind $\mathfrak{h}<$ ind $\mathfrak{k}+\operatorname{dim} \mathfrak{h}^{u}$. Следовательно, в неравенстве (6) равенство не достигается, что противоречит коизотропности подалгебры $\mathfrak{h}$ в $\mathfrak{g}$.

Пусть $(\mathfrak{s}, \mathfrak{k})=\left(\mathfrak{s l}_{n+1}, \mathfrak{g l}_{n}\right)$. Из неравенства (7) следует, что $\mathfrak{h}^{u}=\mathfrak{p}^{u}$, причем $\mathfrak{p}^{u}=k^{n+1}-$ коммутативная алгебра Ли. Пространство $k^{n+1}$ разлагается на два неприводимых подпространства относительно действия $\mathfrak{g l}_{n}, k^{n+1}=k^{n} \oplus k$. Таким образом, $\mathfrak{h}=\mathfrak{g l}_{n} \oplus\left(k^{n} \oplus k\right) \subset \mathfrak{s l}_{n+2}$. Из леммы 3 , п. (а), вытекает, что

$$
\text { ind } \mathfrak{h}=\operatorname{ind}\left(\mathfrak{g l}_{n} \oplus k^{n}\right)+1=1 \text {. }
$$

Получаем, что ind $\mathfrak{h}<$ ind $\mathfrak{k}+\operatorname{dim} \mathfrak{h}^{u}$. Следовательно, в неравенстве (6) равенство не достигается, что противоречит коизотропности подалгебры $\mathfrak{h}$ в $\mathfrak{g}$.

Пусть $\mathfrak{k}$ содержит неприводимые компоненты алгебры $\mathfrak{s}$. Поскольку $\mathfrak{s}-$ классическая простая алгебра Ли или $\mathfrak{s o}_{4}=\mathfrak{s l}_{2} \oplus \mathfrak{s l}_{2}$, то $\mathfrak{k}=\mathfrak{s}$ или $\mathfrak{k}=\mathfrak{s l}_{2} \subset \mathfrak{s}=$ $\mathfrak{s l}_{2} \oplus \mathfrak{s l}_{2}$, где $\mathfrak{k}$ вкладывается в $\mathfrak{s}$ как прямое слагаемое. Во втором случае ind $\mathfrak{h}=3$ (лемма 3 , п. (e)) и неравенство $(*)$ не выполнено, что противоречит коизотропности подалгебры $\mathfrak{h}$ в $\mathfrak{g}=\mathfrak{s o}_{6}$.

Пусть $\mathfrak{h}=\mathfrak{s} \oplus \mathfrak{h}^{u}$. Если $\mathfrak{g}=\mathfrak{s l}_{n+1}, \mathfrak{s o} \mathfrak{o}_{n}$, то $\mathfrak{s}$ неприводимо действует на $\mathfrak{p}^{u}$ и поэтому $\mathfrak{h}^{u}=\mathfrak{p}^{u}$. Если $\mathfrak{g}=\mathfrak{s p}_{2 n}$, то нильпотентный радикал есть сумма своего центра $\mathfrak{c}$ и некоторого неприводимого подпространства $V, \mathfrak{h}^{u}=\mathfrak{c}+V$, 
причем $[V, V]=\mathfrak{c}$. Если $\mathfrak{h}=\mathfrak{s} \oplus \mathfrak{c}$, то $\mathfrak{h}$ не удовлетворяет равенству $(*)$. Следовательно, $\mathfrak{h}=\mathfrak{s} \oplus \mathfrak{p}^{u}$ и $\mathfrak{h}=[\mathfrak{p}, \mathfrak{p}]$. Получаем, что $\mathfrak{h}=\mathfrak{g}_{v}$. Как было показано выше, все такие подалгебры являются коизотропными.

Получаем полный список пар $(\mathfrak{g}, \mathfrak{h})$, где подалгебра $\mathfrak{h}$ имеет полупростую подалгебру Леви и коизотропна: $\left(\mathfrak{s l}_{n},\left(\mathfrak{s l}_{n}\right)_{v}\right),\left(\mathfrak{s o}_{n},\left(\mathfrak{s o}_{n}\right)_{v}\right),\left(\mathfrak{s p}_{n},\left(\mathfrak{s p}_{n}\right)_{v}\right)$.

Пусть теперь $\mathfrak{k} \not \mathfrak{s}$. Тогда $\mathfrak{z k}=\mathfrak{k} \cap \mathfrak{z} \neq 0$ и k не является полупростой. Положим $\mathfrak{k}^{\prime}=[\mathfrak{k}, \mathfrak{k}], \mathfrak{h}^{\prime}=\mathfrak{k}^{\prime} \oplus \mathfrak{h}^{u}$ (вообще говоря, $\mathfrak{h}^{\prime}$ не совпадает с коммутантом алгебры $\mathfrak{h})$. Подалгебра Леви алгебры $\mathfrak{h}^{\prime}$ совпадает с $\mathfrak{k}^{\prime}$ и полупроста.

Пусть $(\mathfrak{g}, \mathfrak{p})=\left(\mathfrak{s o}_{n},\left(\mathfrak{s o}_{n}\right)_{\langle v\rangle}\right)$. Тогда $\mathfrak{z k}=\mathfrak{z}$, поскольку центр $\mathfrak{z}$ одномерен. Будем считать, что $\mathfrak{h}^{u}=\mathfrak{p}^{u}$ Рассмотрим $\left(\mathfrak{h}^{u}\right)^{*}$ как $\mathfrak{k}-$ и $\mathfrak{k}^{\prime}$-модули. Легко видеть, что стабилизаторы $\mathfrak{k}_{\xi}$ и $\mathfrak{k}_{\xi}^{\prime}$ точки $\xi \in\left(\mathfrak{h}^{u}\right)^{*}$ общего положения совпадают. Следовательно, $\operatorname{ind}\left(\mathfrak{k},\left(\mathfrak{h}^{u}\right)^{*}\right)=\operatorname{ind}\left(\mathfrak{k}^{\prime},\left(\mathfrak{h}^{u}\right)^{*}\right)-1$. Таким образом, по теореме Раиса ind $\mathfrak{h}=\operatorname{ind} \mathfrak{h}^{\prime}-1$. Так как $\operatorname{dim} \mathfrak{h}=\operatorname{dim} \mathfrak{h}^{\prime}+1$, то $\operatorname{cork}_{\mathfrak{g}} \mathfrak{h}=\operatorname{cork}_{\mathfrak{g}} \mathfrak{h}^{\prime}$. Значит, подалгебра $\mathfrak{h}^{\prime}$ должна быть коизотропной в $\mathfrak{g}$. Таким образом, $\mathfrak{h}^{\prime}=[\mathfrak{p}, \mathfrak{p}]$ и $\mathfrak{h}=\mathfrak{p}$. Приходим к уже упомянутой паре.

Пусть $(\mathfrak{g}, \mathfrak{p})=\left(\mathfrak{s p}_{n},\left(\mathfrak{s p}_{n}\right)_{\langle v\rangle}\right)$. Тогда $\mathfrak{z}_{\mathfrak{k}}=\mathfrak{z}$, поскольку $\operatorname{dim} \mathfrak{z}=1$. Алгебра $\mathfrak{p}$ имеет вид $\mathfrak{p}=\mathfrak{p}(0) \oplus \mathfrak{p}(1) \oplus \mathfrak{p}(2)$, где $\mathfrak{p}(0)$ - подалгебра Леви, $\mathfrak{p}(1) \oplus \mathfrak{p}(2)-$ унипотентный радикал, $\mathfrak{p}(2)$ - его центр. Алгебра $\mathfrak{h}$ наследует градуировку с градуировки алгебры $\mathfrak{p}$. Будем считать, что $\mathfrak{h}^{u}=\mathfrak{p}^{u}$. Рассмотрим $\mathfrak{h}(2)^{*}$ как $\mathfrak{k}$ - и $\mathfrak{k}^{\prime}$-модули. Стабилизаторы $\mathfrak{k}_{\xi}$ и $\mathfrak{k}_{\xi}^{\prime}$ точки $\xi \in \mathfrak{h}(2)^{*}$ общего положения совпадают. Следовательно, ind $\left(\mathfrak{k}, \mathfrak{h}(2)^{*}\right)=\operatorname{ind}\left(\mathfrak{k}^{\prime}, \mathfrak{h}(2)^{*}\right)-1$. Таким образом, согласно теореме 7 , ind $\mathfrak{h}=$ ind $\mathfrak{h}^{\prime}-1$. Taк $\operatorname{кaK} \operatorname{dim} \mathfrak{h}=\operatorname{dim} \mathfrak{h}^{\prime}+1$, то $\operatorname{cork}_{\mathfrak{g}} \mathfrak{h}=\operatorname{cork}_{\mathfrak{g}} \mathfrak{h}^{\prime}$. Значит, подалгебра $\mathfrak{h}^{\prime}$ должна быть коизотропной в $\mathfrak{g}$. Таким образом, $\mathfrak{h}^{\prime}=[\mathfrak{p}, \mathfrak{p}]$ и $\mathfrak{h}=\mathfrak{p}$. Мы приходим к уже упомянутой выше паре.

Пусть $(\mathfrak{g}, \mathfrak{p})=\left(\mathfrak{s l}_{n+1}, \mathfrak{p}_{\left(\alpha_{1}, \alpha_{n}\right)}\right)$. Пусть $n \geqslant 3$. Как и выше, на алгебре $\mathfrak{h}$ есть градуировка $\mathfrak{h}=\mathfrak{h}(0) \oplus \mathfrak{h}(1) \oplus \mathfrak{h}(2)$. Если $\mathfrak{z} \mathfrak{z} \neq \mathfrak{z}$ и $\mathfrak{z} \mathfrak{k} \neq\left\langle(n-1) E_{11}-2\left(E_{22}+\cdots\right.\right.$ $\left.\left.+E_{n n}\right)+(n-1) E_{n+1, n+1}\right\rangle$, то, рассуждая, как в предыдущем абзаце, получаем, что $\mathfrak{k}_{\xi}=\mathfrak{k}_{\xi}^{\prime}$ и подалгебра $\mathfrak{h}^{\prime}$ коизотропна в $\mathfrak{g}$. Но в алгебре $\mathfrak{p}\left(\alpha_{1}, \alpha_{n}\right)$ не существует коизотропных подалгебр с тривиальной группой характеров. Следовательно, если $n \geqslant 3$, то $\mathfrak{k}=\mathfrak{z}$ или $\mathfrak{z k}=\left\langle(n-1) E_{11}-2\left(E_{22}+\cdots+E_{n n}\right)+(n-1) E_{n+1, n+1}\right\rangle$.

Далее, из неравенства (8) вытекает, что

$$
\operatorname{dim} \mathfrak{s}-\operatorname{dim} \mathfrak{k}^{\prime}-\operatorname{rk} \mathfrak{s}-\operatorname{rk} \mathfrak{k}^{\prime} \leqslant 2 \operatorname{dim} \mathfrak{z} \mathfrak{k} .
$$

Отметим, что правая часть не превосходит 4 и $\mathfrak{s}=\mathfrak{s l}_{n-1}$. Все редуктивные подалгебры алгебры $\mathfrak{s l}_{n-1}$ коранга не более 4 перечислены в $\S 4$. Прямая проверка всех возможных вариантов дает следующий список коизотропных пар, отличных от перечисленных выше: $\left(\mathfrak{s l}_{3}, \mathfrak{b}\right)$ и $\left(\mathfrak{s l}_{3}, \mathfrak{u}+\left\langle E_{11}+2 E_{22}+E_{33}\right\rangle\right)$, где $\mathfrak{b}-$ борелевская подалгебра в $\mathfrak{s l}_{3}$, a $\mathfrak{u}$ - ее унипотентный радикал.

Наконец, пусть $(\mathfrak{g}, \mathfrak{p})=\left(\mathfrak{s l}_{n+1},\left(\mathfrak{s l}_{n+1}\right)_{\langle v\rangle}\right)$. Тогда $\mathfrak{z k}=\mathfrak{z}$, поскольку $\operatorname{dim} \mathfrak{z}=1$. Из неравенства (10) следует, что алгебра $\mathfrak{k}^{\prime}$ есть подалгебра коранга не более 2 в $\mathfrak{s}=\mathfrak{s l}_{n}$. Прямая проверка показывает, что данный случай не дает новых коизотропных пар $(\mathfrak{g}, \mathfrak{h})$. Таким образом, доказана теорема 2.

\section{§6. Нередуктивные коизотропные подалгебры с тривиальной группой характеров в полупростых алгебрах Ли}

Пусть алгебра Ли $\mathfrak{g}=\bigoplus_{i=1}^{m} \mathfrak{g}_{i}$ полупроста, $\mathfrak{g}_{i}$ - ее простые идеалы, а подалгебра $\mathfrak{h}$ нередуктивна, не имеет характеров и не содержит идеалов алгебры $\mathfrak{g}$. 
Зафиксируем разложение Леви $\mathfrak{h}=\mathfrak{k} \oplus \mathfrak{h}^{u}$, где алгебра $\mathfrak{k}$ полупроста. Можно считать, что

$$
\mathfrak{h} \subset \mathfrak{q}=\bigoplus_{i=1}^{k} \mathfrak{q}_{i} \oplus \bigoplus_{j=k+1}^{m} \mathfrak{g}_{j},
$$

где $\mathfrak{q}_{i}-$ коммутант параболической подалгебры $\mathfrak{p}_{i} \subset \mathfrak{g}_{i}$, причем $\mathfrak{h}^{u} \subset \bigoplus_{i=1}^{m} \mathfrak{p}_{i}^{u}$. Пусть $\mathfrak{q}_{i}=\mathfrak{s}_{i} \oplus \mathfrak{p}_{i}^{u}$ - разложение Леви. Алгебра $\mathfrak{s}_{i}$ полупроста, так как алгебра $\mathfrak{h}$ не имеет нетривиальных характеров. Можно считать, что

$$
\mathfrak{k} \subset \mathfrak{s}=\bigoplus_{i=1}^{k} \mathfrak{s}_{i} \oplus \bigoplus_{j=k+1}^{m} \mathfrak{g}_{j} .
$$

Будем считать, что $\mathfrak{s}_{j}=\mathfrak{g}_{j}$ при $k+1 \leqslant j \leqslant m$. Как было отмечено выше, достаточно найти неразложимые коизотропные пары.

Рассмотрим проекцию $\pi_{i}: \mathfrak{g} \rightarrow \mathfrak{g}_{i}$. Подалгебра $\pi_{i}(\mathfrak{h})$ коизотропна в $\mathfrak{g}_{i}$. Следовательно, $\pi_{i}(\mathfrak{h})=\mathfrak{q}_{i}$ и $\pi_{i}(\mathfrak{k})=\mathfrak{s}_{i}$ при $1 \leqslant i \leqslant k$. Если $k+1 \leqslant j \leqslant m$ и $\mathfrak{g}_{j} \neq \mathfrak{s l}_{2}$, то $\pi_{j}(\mathfrak{h})=\mathfrak{g}_{j}$ или $\pi_{j}(\mathfrak{h})=\mathfrak{s o}_{n-1}$, если $\mathfrak{g}_{j}=\mathfrak{s o}_{n}$. Отсюда следует, что если $\mathfrak{s}_{i} \neq \mathfrak{s o}_{4}$, то алгебра $\pi_{i}(\mathfrak{k})$ проста.

Пусть $\mathfrak{s}_{i} \neq \mathfrak{s o}_{4}, \mathfrak{s l}_{2}$. Тогда алгебра $\pi_{i}(\mathfrak{k})$ проста. Следовательно, $\mathfrak{k}=\mathfrak{k}_{i} \oplus$ $\left(\operatorname{Ker} \pi_{i} \cap \mathfrak{k}\right)$, где $\mathfrak{k}_{i} \simeq \pi_{i}(\mathfrak{k})-$ простой идеал алгебры $\mathfrak{k}$, причем $\mathfrak{k}_{i} \neq \mathfrak{s l}_{2}$. Таким образом, если $\pi_{j}(\mathfrak{k}) \neq 0$ для некоторого $j$, то $\pi_{j}\left(\mathfrak{k}_{i}\right)=\pi_{j}(\mathfrak{k})$ и, следовательно, $\pi_{j}\left(\operatorname{Ker} \pi_{i} \cap \mathfrak{k}\right)=0$. Пусть $I-$ множество всех таких $j$, что $\pi_{j}(\mathfrak{k}) \neq 0$. Положим

$$
\mathfrak{s}_{I}=\bigoplus_{i \in I} \mathfrak{s}_{i}, \quad \mathfrak{h}_{I}=\bigoplus_{i \in I} \mathfrak{h}_{i}, \quad \mathfrak{g}_{I}=\bigoplus_{i \in I} \mathfrak{g}_{i} .
$$

Так как $\mathfrak{k}_{i}$ - идеал в $\mathfrak{s}$, то $\mathfrak{h}_{I}-$ идеал в $\mathfrak{h}$. Следовательно, пару $\left(\mathfrak{g}_{I}, \mathfrak{h}_{I}\right)$ можно отщепить. Когда такая пара будет коизотропной? Аналогично тому, как это делалось в $\S 4$, можно установить неравенства (6)-(9). Из неравенства (9) следует, что $\mathfrak{k}_{i}$ является коизотропной подалгеброй в $\mathfrak{s}_{I}$. Из классификации Кнопа следует, что алгебра $\mathfrak{s}_{I}$, а значит, и $\mathfrak{g}_{I}$, должна быть простой. Следовательно, пара $\left(\mathfrak{g}_{i}, \mathfrak{h}_{i}\right)$ есть одна из пар, перечисленных в $\S \S 2$ и 3.

Пусть теперь $\mathfrak{s}_{i}=\mathfrak{s o}_{4}=\mathfrak{s l}_{2} \oplus \mathfrak{s l}_{2}$. Тогда $\mathfrak{g}_{i}=\mathfrak{s o}_{6}$ и $\mathfrak{q}_{i}=\left(\mathfrak{s o}_{6}\right)_{v}$. Имеем $\mathfrak{k}=\mathfrak{k}_{i} \oplus$ $\left(\operatorname{Ker} \pi_{i} \cap \mathfrak{k}\right)$, где $\mathfrak{k}_{i} \simeq \mathfrak{s l}_{2} \oplus \mathfrak{s l}_{2}$. Обозначим простые слагаемые алгебры $\mathfrak{k}_{i}$ через $\mathfrak{k}_{i_{1}}$ и $\mathfrak{k}_{i_{2}}$. Можно считать, что существует такое $j \neq i_{1}$, что $\pi_{j}\left(\mathfrak{k}_{i_{1}}\right) \neq 0$ (иначе пару $\left(\mathfrak{g}_{i}, \mathfrak{h}_{i}\right)$ можно отщепить). Значит, $\mathfrak{k}_{i_{1}}$ вкладывается диагональным образом в некоторые простые слагаемые алгебры $\mathfrak{s}$, изоморфные $\mathfrak{s l}_{2}$. Обозначим два из этих слагаемых через $\mathfrak{s}_{i_{1}}$ и $\mathfrak{s}_{i_{2}}$. При этом будем считать, что $\mathfrak{s}_{i_{1}} \subset \mathfrak{s}_{i}$ и $\mathfrak{s}_{i_{2}} \subset \mathfrak{s}_{j}$, $j \neq i$. Увеличив подалгебру $\mathfrak{h}$, можно считать, что $\mathfrak{k}$ содержит все остальные слагаемые алгебры $\mathfrak{s}$, изоморфные $\mathfrak{s l}_{2}$. Тогда $\mathfrak{k}_{i}-$ идеал в $\mathfrak{s}$, а $\mathfrak{h}_{i}-$ идеал в $\mathfrak{h}$. Следовательно, пару $\left(\mathfrak{g}_{i} \oplus \mathfrak{g}_{j}, \mathfrak{h} \cap\left(\mathfrak{g}_{i} \oplus \mathfrak{g}_{j}\right)\right)$ можно отщепить. Пара $\left(\mathfrak{g}_{j}, \mathfrak{q}_{j}\right)$ может быть одной из следующих: $\left(\mathfrak{s l}_{2}, \mathfrak{s l}_{2}\right),\left(\mathfrak{s l}_{3},\left(\mathfrak{s l}_{3}\right)_{v}\right),\left(\mathfrak{s o}_{6},\left(\mathfrak{s o}_{6}\right)_{v}\right)$. Прямая проверка показывает, что ни в одной алгебре $\mathfrak{g}_{i} \oplus \mathfrak{g}_{j}$ нет неразложимых коизотропных подалгебр, кроме случая $\mathfrak{s l}_{2} \subset \mathfrak{s o}_{4}$, указанного выше. Поэтому компонента $\mathfrak{k}_{i_{1}}$ не может вкладываться диагонально в $\mathfrak{s}$ и пара $(\mathfrak{g}, \mathfrak{h})$ является разложимой.

Пусть $\mathfrak{s}_{i}=\mathfrak{s l}_{2}$. Тогда $\mathfrak{k}=\mathfrak{k}_{i} \oplus\left(\operatorname{Ker} \pi_{i} \cap \mathfrak{k}\right)$, где $\mathfrak{k}_{i} \simeq \mathfrak{s l}_{2}$. Алгебра $\mathfrak{k}_{i}$ может вкладываться диагональным образом в несколько компонент алгебры $\mathfrak{s}$, изоморфных $\mathfrak{s l}_{2}$. Как и в предыдущем абзаце, можно считать, что $\mathfrak{k}_{i}$ вкладывается 
только в две компоненты $\mathfrak{s}_{i_{1}}$ и $\mathfrak{s}_{i_{2}}$, причем $\mathfrak{s}_{i_{1}} \subset \mathfrak{s}_{i}, \mathfrak{s}_{i_{2}} \subset \mathfrak{s}_{j}, i \neq j$. Следовательно, пару $\left(\mathfrak{g}_{i} \oplus \mathfrak{g}_{j}, \mathfrak{h} \cap\left(\mathfrak{g}_{i} \oplus \mathfrak{g}_{j}\right)\right)$ можно отщепить. Прямая проверка показывает, что среди таких пар также нет неразложимых коизотропных.

Таким образом, доказана теорема 3.

\section{§7. Структура централизаторов коизотропных подалгебр в универсальной обертывающей алгебре}

В этом параграфе мы докажем теорему 4, сформулированную во введении. В случае когда $\mathfrak{h}$ - редуктивная подалгебра, она была доказана Кнопом [1]. Поэтому далее мы будем считать, что пара $(\mathfrak{g}, \mathfrak{h})$ - одна из пар $\left(\mathfrak{g}, \mathfrak{g}_{v}\right)$, перечисленных в п. 2) теоремы 3 .

Зафиксируем следующие базисы и инвариантные билинейные формы $\alpha$ в пространстве $V$ стандартного представления $\rho$ классической простой алгебры $\mathfrak{g}$, а также максимальный тор $\mathfrak{t} \subset \mathfrak{g}:$

1) $\mathfrak{g}=\mathfrak{s l}_{n+1}$, базис $\left\{e_{1}, \ldots, e_{n+1}\right\}$, максимальный тор $\mathfrak{t}=\operatorname{diag}\left(t_{1}, \ldots, t_{n},-t_{1}-\right.$ $\left.\cdots-t_{n}\right)$;

2) $\mathfrak{g}=\mathfrak{s p}_{2 n}$, базис $\left\{e_{1}, \ldots, e_{n}, e_{-n}, \ldots, e_{-1}\right\}$, билинейная форма $\alpha(x, y)=$ $x_{1} y_{-1}-y_{1} x_{-1}+\cdots+x_{n} y_{-n}-y_{n} x_{-n}$, максимальный тор $\mathfrak{t}=\operatorname{diag}\left(t_{1}, \ldots, t_{n},-t_{-n}\right.$, $\left.\ldots,-t_{-1}\right)$;

3) $\mathfrak{g}=\mathfrak{s o}_{2 n}$, базис $\left\{e_{1}, \ldots, e_{n}, e_{-n}, \ldots, e_{-1}\right\}$, билинейная форма $\alpha(x, y)=$ $x_{1} y_{-1}+x_{-1} y_{1}+\cdots+x_{n} y_{-n}+x_{-n} y_{n}$, максимальный тор $\mathfrak{t}=\operatorname{diag}\left(t_{1}, \ldots, t_{n},-t_{-n}\right.$, $\left.\ldots,-t_{-1}\right)$;

4) $\mathfrak{g}=\mathfrak{s o}_{2 n+1}$, базис $\left\{e_{1}, \ldots, e_{n}, e_{0}, e_{-n}, \ldots, e_{-1}\right\}$, билинейная форма $\alpha(x, y)=$ $x_{1} y_{-1}+x_{-1} y_{1}+\cdots+x_{n} y_{-n}+x_{-n} y_{n}$, максимальный тор $\mathfrak{t}=\operatorname{diag}\left(t_{1}, \ldots, t_{n}, 0,-t_{-n}\right.$, $\left.\ldots,-t_{-1}\right)$.

Будем считать, что вектор $v$ - это $e_{1}$, первый базисный вектор в указанных базисах. Также положим $q(x)=\alpha(x, x)$.

Зафиксируем борелевскую подалгебру верхнетреугольных матриц $\mathfrak{b} \subset \mathfrak{g}$, стабилизирующую прямую $\langle v\rangle$ и содержащую тор $\mathfrak{t}$. Обозначим унипотентный радикал алгебры $\mathfrak{b}$ через $\mathfrak{u}$, а стабилизатор вектора $v$ в $\mathfrak{t}$ через $\mathfrak{t}_{0}$. Алгебра $\mathfrak{t}_{0} \oplus \mathfrak{u}$ есть борелевская подалгебра в $\mathfrak{h}$. Пусть П - множество простых корней относительно $\mathfrak{t}$, такое, что алгебра $\mathfrak{b}$ есть сумма максимального тора $\mathfrak{t}$ и корневых подпространств, отвечающих положительным корням. Старший вес стандартного представления относительно $\mathfrak{t}$ обозначим через $\lambda_{1}$, так что $\lambda_{1}\left(\mathfrak{t}_{0}\right)=0$. Через $\Lambda_{1}=l \lambda_{1}, l \in \mathbb{Z}_{+}$, обозначим наименьший вес, кратный $\lambda_{1}$ и лежащий в $\mathbb{Z}_{+}$П. Легко видеть, что $\Lambda_{1}=(n+1) \lambda_{1}$ для алгебры $\mathfrak{s l}_{n+1}, \Lambda_{1}=\lambda_{1}$ для алгебры $\mathfrak{s o}_{2 n+1}$ и $\Lambda_{1}=2 \lambda_{1}$ для алгебр $\mathfrak{s o}_{2 n}$ и $\mathfrak{s p}_{2 n}$.

Будем обозначать через $\rho(\mu): \mathfrak{g} \rightarrow \mathfrak{g l}(V(\mu))$ неприводимое представление алгебры $\mathfrak{g}$ со старшим весом $\mu$ в пространстве $V(\mu)$. Рассмотрим симметрическую степень $\rho_{m}=S^{l m}(\rho)$ стандартного представления $\rho=\rho\left(\lambda_{1}\right)$. В случаях $\mathfrak{s l}_{n+1}$ и $\mathfrak{s p}_{2 n}$ представление $\rho_{m}$ совпадает с представлением $\rho\left(m \Lambda_{1}\right)$. В случае $\mathfrak{s o}_{2 n}$ имеем $\rho_{m}=\rho\left(m \Lambda_{1}\right) \oplus q \rho_{m-1}$. В случае $\mathfrak{s o}_{2 n+1}$ имеем $\rho_{m}=\rho\left(m \Lambda_{1}\right) \oplus q \rho_{m-2}$. Легко видеть, что справедлива следующая

Лемма 4. Подпространство веса 0 относителъно тора $\mathfrak{t}$ в пространстве $V_{m}$ представления $\rho_{m}$ имеет следующий вид:

1) $\left\langle\left(x_{1} x_{2} \ldots x_{n+1}\right)^{m}\right\rangle$ в случае $\mathfrak{s l}_{n+1}$;

2) линейная оболочка мономов степени $m$, являющихся произведениями мономов $x_{0}, x_{1} x_{-1}, \ldots, x_{n-1} x_{-(n-1)}$, в случае $\mathfrak{s o}_{2 n+1}$; 
3) многочлены степени $2 m$, порождаемые мономами $x_{1} x_{-1}, \ldots, x_{n-1} x_{-(n-1)}$, в случаях $\mathfrak{s p}_{2 n} u \mathfrak{s o}_{2 n}$.

Следующая лемма описывает строение центра $Z(\mathfrak{h})$ алгебры $P(\mathfrak{h})$.

Лемма 5. Алгебра $Z(\mathfrak{h})$ есть алгебра многочленов $k\left[f_{1}, \ldots, f_{d}\right]$ om $d=$ ind $\mathfrak{h}$ переменных. Более того,

1) в случае $\mathfrak{g}=\mathfrak{s l}_{n+1}, \mathfrak{s o}_{2 n}, \mathfrak{s p}_{2 n}$ можно выбрать порождающие $f_{1}, \ldots, f_{d}$ так, что они имеют вес $\Lambda_{1}$ относительно максимального тора $\mathfrak{t} \subset \mathfrak{g}$;

2) в случае $\mathfrak{g}=\mathfrak{s o}_{2 n+1}$ можно выбрать порождающие $f_{1}, \ldots, f_{d}$ так, что $f_{1}$ имеет вес $\Lambda_{1}$, a $f_{2}, \ldots, f_{d}$ имеют вес $2 \Lambda_{1}$ относительно $\mathfrak{t}$.

Доказательство. Пусть $\mathfrak{g}=\mathfrak{s l}_{n+1}$. Тогда $\mathfrak{h}=\mathfrak{s l}_{n} \oplus k^{n}$. Пусть $(A, v) \in \mathfrak{h}, A \in$ $\mathfrak{s l}_{n}, v \in k^{n}$. Легко видеть, что многочлен $\operatorname{det}\left(v, A v, A^{2} v, \ldots, A^{n-1} v\right)$ является инвариантом и его вес относительно тора равен $\Lambda_{1}$.

Если $\mathfrak{g}=\mathfrak{s o}_{r}$, то алгебра $\mathfrak{h}=\mathfrak{s o}_{r-2} \oplus k^{r-2}$ есть $\mathbb{Z}_{2}$-стягивание алгебры $\hat{\mathfrak{h}}=\mathfrak{s o}_{r-1}[12]$. Точнее, зафиксируем координаты $x_{1}^{\prime}, \ldots, x_{r-1}^{\prime}$ и форму $\alpha^{\prime}\left(x^{\prime}\right)=$ $x_{1}^{\prime 2}+\cdots+x_{r-1}^{2}$ в пространстве стандартного представления алгебры $\hat{\mathfrak{h}}$. Рассмотрим элемент $t=\operatorname{diag}(1, \ldots, 1,-1)$, и пусть $\theta-$ сопряжение элементом $t$. Это инволютивный автоморфизм алгебры $\hat{\mathfrak{h}}$. Он задает $\mathbb{Z}_{2}$-градуировку на $\hat{\mathfrak{h}}=\mathfrak{h}_{0} \oplus \mathfrak{h}_{1}$, где $\mathfrak{h}_{0}=\mathfrak{s o}_{r-2}$ и $\mathfrak{h}_{1}=V, V=k^{r-2}$. Зададим на пространстве $\mathfrak{h}$ новое умножение $[\cdot, \cdot]^{\prime}$. Положим $\left[\mathfrak{h}_{0}, \mathfrak{h}_{i}\right]^{\prime}=\left[\mathfrak{h}_{0}, \mathfrak{h}_{i}\right], i=0,1$, и $\left[\mathfrak{h}_{1}, \mathfrak{h}_{1}\right]^{\prime}=0$. Алгебра с таким умножением есть в точности $\mathfrak{h}$. Известно [12], что в этом случае алгебра $Z(\mathfrak{h})$ свободна и что порождающие инварианты алгебры $\mathfrak{h}$ можно получить из инвариантов алгебры $\hat{\mathfrak{h}}$.

Пусть $\hat{f}_{1}, \ldots, \hat{f}_{d}$ - образующие алгебры инвариантов алгебры $\mathfrak{s o}_{r-1}$. Представим их в виде $\hat{f}_{i}=\bigoplus h_{j} v_{j}$, где $h_{j} \in k\left[\mathfrak{s o}_{r-2}\right], v_{j} \in k[V]$. Алгебра $Z(\mathfrak{g})$ порождается инвариантами $f_{i}$, где $f_{i}$ есть сумма тех мономов многочлена $\hat{f}_{i}$, для которых степень множителя $v_{j}$ максимальна. В случае когда $\mathfrak{g}=\mathfrak{s o}_{2 n}$, алгебра $\mathfrak{h}$ получается стягиванием алгебры $\mathfrak{s o}_{2 n-1}$. Инварианты этой алгебры суть суммы главных миноров четных порядков. Следовательно, инварианты алгебры $\mathfrak{h}$ - суммы главных миноров четных порядков, в которые входит первый столбец и первая строка. Легко видеть, что максимальная степень мономов по $V$ равна 2 и вес относительно тора $\mathfrak{t}$ есть $\Lambda_{1}$.

Аналогично, в случае $\mathfrak{g}=\mathfrak{s o}_{2 n+1}$ алгебра $\mathfrak{h}$ получается стягиванием алгебры $\mathfrak{s o}_{2 n}$. Инварианты этой алгебры порождаются суммами главных миноров четного порядка и пфаффианом. Соответственно максимальные степени по $V$ равны 2 и 1 , а веса относительно тора $\mathfrak{t}$ равны $2 \Lambda_{1}$ и $\Lambda_{1}$.

В случае $\mathfrak{g}=\mathfrak{s p}_{2 n}$ алгебра $\mathfrak{h}$ имеет одномерный центр. Обозначим порождающую центра через $е$. При этом $\mathfrak{h}=\mathfrak{g}_{e}$ - централизатор нильпотентного элемента $e$ в $\mathfrak{g}$. В [13] доказано, что алгебра $Z\left(\mathfrak{g}_{e}\right)$ свободна. Более того, там же описана процедура получения порождающих алгебры $Z\left(\mathfrak{g}_{e}\right)$ из порождающих алгебры $Z(\mathfrak{g})$. В данном случае процедуру получения порождающих алгебры $Z(\mathfrak{h})$ можно описать следующим образом. Пусть $f$ - нильпотентный элемент, противоположный элементу $e$ относительно тора $\mathfrak{t}$, и $\left\{\hat{F}_{i}\right\}, 1 \leqslant i \leqslant n$, - система порождающих алгебры $Z\left(\mathfrak{s p}_{2 n}\right)$, а именно, $F_{i}$ - сумма определителей главных миноров порядка $2 i$. Рассмотрим многочлен $\overline{F_{i}}\left(\mathfrak{g}_{e}\right)=\hat{F}_{i}\left(f+\mathfrak{g}_{e}\right)$ и возьмем однородную компоненту $F_{i}$ многочлена $\overline{F_{i}}$ наименьшей степени. Многочлены $F_{i}$ 
являются порождающими алгебры h. Легко видеть, что веса порождающих $F_{i}$ относительно тора $\mathfrak{t}$ равны $\Lambda_{1}$.

Замечание. Поскольку $Z(\mathfrak{g})=\operatorname{gr} \mathfrak{Z}(\mathfrak{g})$ и отображение симметризации $\psi$ является поднятием, алгебра $\mathfrak{Z}(\mathfrak{g})$ порождается элементами $\psi\left(f_{1}\right), \ldots, \psi\left(f_{d}\right)$.

Пусть $G$ - алгебраическая группа с касательной алгеброй $\mathfrak{g}$, a $H \subset G-$ алгебраическая подгруппа с касательной алгеброй $\mathfrak{h}$.

Лемма 6. Пусть $M_{0}$ - нуль-конус коприсоединенного представления $G: \mathfrak{g}^{*}$. Действие $H: M_{0}$ имеет конечный стабилизатор общего положения.

Доказательство. Будем считать, что $G$ - классическая простая группа Ли. Пространство стандартного представления группы $G$ будем также обозначать через $V$ и зафиксируем там такой же базис, как и для соответствующего представления алгебры g. Будем считать, что $H=G_{v}$.

1) Случай $\mathfrak{g}=\mathfrak{s l}_{n+1}$. Рассмотрим точку

$$
x=\left(\begin{array}{ccccc}
0 & 0 & \ldots & 0 \\
1 & 0 & \ldots & 0 \\
\ldots & \ldots & \ldots & \ldots & 0 \\
\ldots & \ldots & 1 & 0 & 0 \\
0 & \ldots & 1 & 0
\end{array}\right) \in M_{0} .
$$

Стабилизатор точки $x$ тривиален. Действительно, пусть $h \in H$ и $h x h^{-1}=x$. Обозначим столбцы матрицы $h$ через $h_{1}, \ldots, h_{n+1}$, при этом $h_{1}=e_{1}$. Столбцы матрицы $h x$ равны $h_{2}, \ldots, h_{n+1}, 0$, поэтому первый столбец матрицы $h x h^{-1}$ равен $h_{2}$ и, следовательно, $h_{2}=e_{2}$. Аналогичным образом последовательно получаем, что $h_{i}=e_{i}$, т. е. $h=E$.

Для других классических алгебр Ли выберем следующие точки $x$ :

2 ) Случай $\mathfrak{g}=\mathfrak{s p}_{2 n}$. Матрица $x=\left(x_{i j}\right), 1 \leqslant i, j \leqslant 2 n$, такая, что $x_{i, i-1}=1$, $2 \leqslant i \leqslant n+1, x_{i, i-1}=-1, n+2 \leqslant i \leqslant 2 n, x_{i j}=0, i-j \neq 1$.

3) Случай $\mathfrak{g}=\mathfrak{s o}_{2 n+1}$. Матрица $x=\left(x_{i j}\right), 1 \leqslant i, j \leqslant 2 n+1$, такая, что $x_{i, i-1}=1,2 \leqslant i \leqslant n+1, x_{i, i-1}=-1, n+2 \leqslant i \leqslant 2 n+1, x_{i j}=0, i-j \neq 1$.

4) Случай $\mathfrak{g}=\mathfrak{s o}_{2 n+1}$. Матрица $x=\left(x_{i j}\right), 1 \leqslant i, j \leqslant 2 n$, такая, что $x_{i, i-1}=1$, $2 \leqslant i \leqslant n, x_{i, i-1}=-1, n+2 \leqslant i \leqslant 2 n, x_{n+1, n-1}=1, x_{n+2, n}=-1$ и $x_{i j}=0$ в остальных случаях.

Тривиальность стабилизатора точки $x$ проверяется аналогично случаю $\mathfrak{s l}_{n+1}$.

Обозначим через $Z(\mathfrak{g})_{+}$множество элементов из $Z(\mathfrak{g})$ с нулевым свободным членом и рассмотрим идеал $Z(\mathfrak{g})_{+} P(\mathfrak{g})$ в $P(\mathfrak{g})$.

Следствие. $Z(\mathfrak{g})_{+} P(\mathfrak{g}) \cap P(\mathfrak{h})=0$.

Доказательство. Поскольку группа $H$ имеет конечный стабилизатор общего положения при действии на нуль-конусе $M_{0}$ (лемма 6), отображение моментов $\Phi: M_{0} \rightarrow \mathfrak{h}^{*}$ доминантно и, следовательно, соответствующее отображение алгебр функций $\Phi^{*}: P(\mathfrak{h}) \rightarrow k\left[M_{0}\right]$ инъективно. Так как $k\left[M_{0}\right]=P(\mathfrak{g}) / I\left(M_{0}\right)$, где $I\left(M_{0}\right)=Z(\mathfrak{g})_{+} P(\mathfrak{g})$ - идеал нулей нуль-конуса $M_{0}$, то $Z(\mathfrak{g})_{+} P(\mathfrak{g}) \cap P(\mathfrak{h})$ $=0$.

Доказательство теоремы 4. 1) Если $A$ - однородное дополнение к идеалу $Z(\mathfrak{g})_{+} P(\mathfrak{g})$ в $P(\mathfrak{g})$, то $P(\mathfrak{g}) \simeq A \otimes Z(\mathfrak{g})$ (как векторное пространство), причем изоморфизм задается перемножением компонент [14, теорема 0.2]. Можно выбрать 
подпространство $A$ так, что оно будет инвариантным относительно присоединенного действия алгебры $\mathfrak{g}\left[14\right.$, теорема 0.9]; тогда $P(\mathfrak{g})^{\mathfrak{h}} \simeq A^{\mathfrak{h}} \otimes Z(\mathfrak{g})$.

Из леммы 1 следует, что отображение моментов $\Phi: M \rightarrow \mathfrak{h}^{*}$ доминантно для симплектического слоя общего положения $M$, так что $P(\mathfrak{h}) \hookrightarrow k[M]$. Следовательно,

$$
P(\mathfrak{h}) \otimes Z(\mathfrak{g}) \hookrightarrow P(\mathfrak{g}) .
$$

Действительно, предположим, что существуют линейно независимые $f_{1}, \ldots, f_{l} \in$ $P(\mathfrak{h})$ и $z_{1}, \ldots, z_{l} \in Z(\mathfrak{g})$, такие, что $z_{1} f_{1}+\cdots+z_{l} f_{l}=0$. Функции $z_{1}, \ldots, z_{l}$ постоянны на слое $M$. Обозначим через $c_{1}, \ldots, c_{l}$ ограничения этих функций на $M$. Получаем, что на $M$ выполнено равенство $c_{1} f_{1}+\cdots+c_{l} f_{l}=0$. Следовательно, $z_{1}=\cdots=z_{l}=0$ на слое $M$ общего положения, а значит, и всюду. Переходя в (11) к h-инвариантам, получаем, что $Z(\mathfrak{h}) \otimes Z(\mathfrak{g}) \hookrightarrow P(\mathfrak{g})^{\mathfrak{h}}$.

Рассмотрим идеалы $I_{1}=Z(\mathfrak{h}) \otimes Z(\mathfrak{g})_{+} \triangleleft Z(\mathfrak{h}) \otimes Z(\mathfrak{g})$ и $I_{2}=P(\mathfrak{g})^{\mathfrak{h}} Z(\mathfrak{g})_{+} \triangleleft P(\mathfrak{g})^{\mathfrak{h}}$, порожденные множеством $Z(\mathfrak{g})_{+}$. По следствию из леммы $6 Z(\mathfrak{h}) \cap I_{2}=0$. Таким образом, $(Z(\mathfrak{h}) \otimes Z(\mathfrak{g})) / I_{1} \hookrightarrow P(\mathfrak{g})^{\mathfrak{h}} / I_{2}$. Алгебра в левой части изоморфна $Z(\mathfrak{h})$, а в правой $A^{\mathfrak{h}}$ (как линейные пространства и даже как $\mathfrak{t}$-модули), причем вложение эквивариантно относительно действия $\mathfrak{t}$. Из [14, теорема 0.9] следует, что кратность вхождения неприводимого представления $\rho(\mu)$ в представление $\mathfrak{g}: A$ равна кратности нулевого веса в пространстве $V(\mu)$. Так как $\mathfrak{t}_{0} \oplus \mathfrak{u} \subset \mathfrak{h}$, то $A^{\mathfrak{h}}$ есть линейная оболочка старших векторов тех представлений $\rho(\mu)$, для которых $\mu\left(\mathfrak{t}_{0}\right)=0$. Следовательно, вес $\mu$ пропорционален весу $\lambda_{1}$ и, более того, весу $\Lambda_{1}$, так как $\mu \in \mathbb{Z}_{+} \Pi$ (иначе в пространстве $V(\mu)$ подпространство нулевого веса тривиально). Таким образом, пространство $A^{\mathfrak{h}}$ как $\mathfrak{t}$-модуль есть сумма подмодулей веса $m \Lambda_{1}$, которые входят в $A^{\mathfrak{h}}$ с кратностью, равной размерности подпространства веса 0 в $\mathfrak{g}$-модуле $V\left(m \Lambda_{1}\right)$. Пространство $Z(\mathfrak{g})$ также является t-модулем. Леммы 4 и 5 дают описание размерностей весовых подпространств $\mathfrak{t}$-модулей $A^{\mathfrak{h}}$ и $Z(\mathfrak{g})$. Легко видеть, что размерности весовых подпространств совпадают и, следовательно, $Z(\mathfrak{h})=A^{\mathfrak{h}}$. Таким образом, все указанные вложения являются равенствами и $P(\mathfrak{g})^{\mathfrak{h}}=Z(\mathfrak{h}) \otimes Z(\mathfrak{g})$.

2) Докажем индукцией по $n$, что $\mathfrak{U}^{(n)}(\mathfrak{g})^{\mathfrak{h}} \subset \mathfrak{Z}(\mathfrak{h}) \otimes \mathfrak{Z}(\mathfrak{g})$. Ясно, что $\mathfrak{U}^{(0)}(\mathfrak{g})^{\mathfrak{h}}=$ $k \subset \mathfrak{Z}(\mathfrak{h}) \otimes \mathfrak{Z}(\mathfrak{g})$. Пусть $\mathfrak{U}^{(n-1)}(\mathfrak{g})^{\mathfrak{h}} \subset \mathfrak{Z}(\mathfrak{h}) \otimes \mathfrak{Z}(\mathfrak{g})$. Пусть $\xi \in \mathfrak{U}^{(n)}(\mathfrak{g})^{\mathfrak{h}}, \bar{\xi}-$ старшая компонента элемента $\xi$ степени $n$ при проекции на $P(\mathfrak{g})^{\mathfrak{h}}$. По п. 1) $\bar{\xi}=\bigoplus_{i=1}^{n} a_{i} b_{i}$, где $a_{i} \in Z(\mathfrak{h}), b_{i} \in Z(\mathfrak{g})$. Рассмотрим некоторые прообразы $\hat{a}_{i} \in \mathfrak{Z}(\mathfrak{h}), \hat{b}_{i} \in \mathfrak{Z}(\mathfrak{g})$ элементов $a_{i}, b_{i}$ в алгебре $\mathfrak{U}(\mathfrak{g})$. Так как $\xi_{n-1}=\xi-\bigoplus_{i=1}^{n} \hat{a}_{i} \hat{b}_{i} \in \mathfrak{U}^{(n-1)}(\mathfrak{g})^{\mathfrak{h}} \subset$ $\mathfrak{Z}(\mathfrak{h}) \otimes \mathfrak{Z}(\mathfrak{g})$, то и $\xi \in \mathfrak{Z}(\mathfrak{h}) \otimes \mathfrak{Z}(\mathfrak{g})$, что и требовалось. Коммутативность алгебры $\mathfrak{U}(\mathfrak{g})^{\mathfrak{h}}$ следует отсюда очевидным образом.

Автор пользуется случаем выразить искреннюю признательность своему научному руководителю Э. Б. Винбергу.

\section{ЛитЕРАТУРА}

[1] F. Knop, Der Zentralisator einer Liealgebra in einer einhullenden Algebra, J. Reine Angew. Math., 406 (1990), 5-9.

[2] Ж. Диксмье, Универсалъные обертывающие алгебры, Мир, М., 1978.

[3] Э. Б. Винберг, Коммутативные однородные пространства и коизотропные симплектические действия, УМН, 56:1 (2001), 3-62. 
[4] Э. Б. Винберг, В. Л. Попов, Теория инвариантов, в кн.: Итоги науки и техники. Современные проблемы математики. Фундаментальные направления, т. 55, ВИНИТИ, М., 1989, 137-309.

[5] D. I. Panyushev, Inductive formulas for the index of seaweed Lie algebras, Moscow Math. J., 1:2 (2001), 221-241.

[6] D. Luna, Sur les orbites fermées des groupes algébriques reductifs, Invent. Math., 16 (1972), 1-5.

[7] P. Tauvel, R. W. T. Yu, Sur l'indice de certaines algèbres de Lie, Ann. Inst. Fourier (Grenoble), 54:6 (2004), 1793-1810.

[8] А. Г. Элашвили, Индекс оросферических подалгебр полупростых алгебр Ли, Труды Тбилисского матем. ин-та, 77 (1985), 116-126.

[9] M. Rais, L'indice des produits semi-directs $E \times{ }_{\rho} \mathfrak{g}$, C. R. Acad. Sci. Paris, Ser A, 287:4 (1978), 195-197.

[10] Е. М. Андреев, Э. Б. Винберг, А. Г. Элашвили, Орбитъ наиболъшей размерности полупростых линейных групп Ли, Функц. анализ и его прил., 1:4 (1967), 3-7.

[11] Дж. Хамфри, Линейные алгебраические группъ, Наука, М., 1980.

[12] D. Panyushev, On the coadjoint representation of $\mathbb{Z}_{2}$-contractions of reductive algebras, Adv. Math., 213:1 (2007), 380-404.

[13] D. Panyushev, A. Premet, O. Yakimova, On symmetric invariants of centralisers in reductive Lie algebras, J. Algebra, 313:1 (2007), 343-391.

[14] B. Kostant, Lie group representations on polynomial ring, Amer. J. Math., 85 (1963), $327-404$.

Московский государственный университет

Поступило в редакцию e-mail: zorin@mccme.ru 13 июля 2007 г. 